\title{
Near-IR spectra of ISOGAL sources in the inner Galactic Bulge ${ }^{\star, \star \star}$
}

\author{
M. Schultheis ${ }^{1}$, A. Lançon ${ }^{2}$, A. Omont ${ }^{1}$, F. Schuller ${ }^{1}$, and D. K. Ojha ${ }^{3}$ \\ ${ }^{1}$ Institut d'Astrophysique de Paris, CNRS, 98bis Bd Arago, 75014 Paris, France \\ 2 Observatoire de Strasbourg, UMR 7550, 11 rue de l'Université, 6700 Strasbourg, France \\ 3 Tata Institute of Fundamental Research, Homi Bhabha Road, Colaba, Mumbai 400 005, India \\ Received 26 June 2002 / Accepted 20 March 2003
}

\begin{abstract}
We present near-IR spectra ( $H K$-band) of a sample of 107 sources with mid-IR excesses at 7 and $15 \mu$ m detected during the ISOGAL survey. Making use of the DENIS interstellar extinction map from Schultheis et al. (1999) we derive luminosities and find that the $M_{\text {bol }}$ vs. ${ }^{12} \mathrm{CO}$ and $M_{\text {bol }}$ vs. $\mathrm{H}_{2} \mathrm{O}$ diagrams are powerful tools for identifying supergiants, AGB stars, giants and young stellar objects. The majority of our sample are AGB stars $(\sim 80 \%)$ while we find four good supergiant candidates, nine young stellar objects and 12 RGB candidates. We have used the most recent $K_{0}-[15]$ relation by Jeong et al. (2002) based on recent theoretical modeling of dust formation of AGB stars to determine mass-loss rates. The mass-loss rates of the supergiants are comparable with those in the solar neighbourhood while the long-period variables cover a mass-loss range from $-5<\log \dot{M}<-7$. The red giant candidates lie at the lower end of the mass-loss rate range between $-6.5<\log \dot{M}<-9$. We used the equivalent width of the $\mathrm{CO}$ bandhead at $2.3 \mu \mathrm{m}$, the NaI doublet and the CaI triplet to estimate metallicities using the relation by Ramírez et al. (2000b). The metallicity distribution of the ISOGAL objects shows a mean $[\mathrm{Fe} / \mathrm{H}] \sim-0.25 \mathrm{dex}$ with a dispersion of \pm 0.40 dex which is in agreement with the values of Ramírez et al. (2000b) for Galactic Bulge fields between $b=-4^{\circ}$ and $b=-1.3^{\circ}$. A comparison with the solar neighbourhood sample of Lançon \& Wood (2000) shows that our sample is $\sim 0.5$ dex more metal-rich on average.
\end{abstract}

Key words. infrared: stars - ISM: dust, extinction - Galaxy: bulge - stars: AGB and post-AGB

\section{Introduction}

The inner galactic Bulge sometimes referred to as the central stellar cluster (see e.g. Serabyn \& Morris 1996), or as the nuclear Bulge (Mezger et al. 1996), presents quite extreme conditions (see also Philipp et al. 1999; Figer 2002). Extending only $\sim 200-300 \mathrm{pc}$ in the galactic plane and $\sim 30-50 \mathrm{pc}$ perpendicular to it, it contains a mass $\sim 4 \times 10^{9} M_{\odot}$, with mean stellar and interstellar densities $\sim 500$ times larger than in the galactic disk. The galactic Bulge provides a wide metallicity range with $-1<[\mathrm{Fe} / \mathrm{H}]<1$ which makes it an ideal place for studying stellar evolution. While in the past, several studies claimed that we deal in the Bulge with a supersolar metal-rich stellar population (see e.g. Whitford 1978; Frogel \& Whitford 1987; Frogel 1988; Rich 1988), chemical abundances there have recently been revised (see e.g. McWilliam \& Rich 1994; Frogel et al. 1999) and at present the iron relative abundance is believed to peak at $\sim-0.3$ dex (Freeman \& Bland-Hawthorn 2003;

Send offprint requests to: $\mathrm{M}$. Schultheis, e-mail: schulthe@iap.fr

* Based on observations collected at the European Southern Observatory, La Silla, Chile.

$\star \star$ The spectra are also available in electronic form at the CDS via anonymous ftp to cdsarc.u-strasbg.fr $(130.79 .128 .5)$ or via http://cdsweb.u-strasbg.fr/cgi-bin/qcat?/A+A/405/531 van Loon et al. 2003; Ibata \& Gilmore 1995; Minniti et al. 1995; Houdashelt 1996), with a wide dispersion. The metallicity distribution is an important ingredient for the different scenarios of galaxy formation such as dissipational collapse or accretion of matter. We want to refer to Freeman \& Hawthorn (2003) for the most recent review about the formation and evolution of our Galaxy.

In most parts of the galactic Bulge, the study of the stellar population is hampered by its high interstellar absorption (Frogel et al. 1999; Schultheis et al. 1999); studies in the Infrared are therefore crucial.

Surveys with the ISO satellite, especially with the ISOCAM instrument (Césarsky et al. 1996), whose sensitivity is several orders of magnitude greater than IRAS and whose angular resolution is ten times better, have led to new possibilities. The ISOGAL 7 and $15 \mu \mathrm{m}$ survey (Omont et al. 2003) in particular has observed $\sim 16 \mathrm{deg}^{2}$ of the central obscured regions of the Galaxy. The total number of stars detected $\left(\sim 10^{5}\right)$ is comparable to the number detected by IRAS in the whole Galaxy. The main goals of the ISOGAL survey are to quantify the spatial distributions of the various stellar populations and their properties in the inner Galaxy, together with the properties of the warm interstellar medium. The combination of ISOGAL and DENIS (or 2MASS) near-infrared 
data is a powerful means for determining the nature of sources even in regions of high extinction ( $A_{V}$ up to 20-30). The various colour-colour and colour-magnitude diagrams available with the five ISOGAL-DENIS bands provide rich information on extinctions, distances, intrinsic colours and absolute magnitudes. Among the $\mathrm{M}$ giants, a large proportion of those detected at $15 \mu \mathrm{m}$ are AGB-LPVs with mass-loss, well traced by their $15 \mu \mathrm{m}$ excesses (Omont et al. 1999; Glass et al. 1999; Alard et al. 2001). Ojha et al. (2003, hereafter referred to as OOS) studied ISOGAL sources in the outer Bulge $\left(|b| \gtrsim 1^{\circ}\right)$ and discussed their nature as well as their mass-loss rates. AGB stars contribute more than $70 \%$ towards the replenishment of the ISM in the solar neighbourhood (Sedlmayr 1994). Thus, it is important to study the mass-loss of these objects in different galactic environments.

However, detailed characterization of the sources faces various difficulties: very large interstellar extinction, uncertainty in the mid-IR extinction law, photometry uncertainty in the case of the weakest ISOGAL sources, etc. Spectroscopic follow-up observations have therefore been deemed essential. For example, Schultheis et al. (2002) obtained visible spectra of nearby sources with mid-IR excesses and could identify interesting objects such as Ae/Be stars, possibly post-AGB stars and stars with red excess. Optical spectroscopy in the Inner Galactic Bulge fails as the sources become invisible due to the high interstellar extinction $\left(A_{V}>20 \mathrm{mag}\right)$. Therefore, only spectroscopy in the near-IR, where interstellar absorption is about ten times smaller (at $\sim 2.2 \mu \mathrm{m}$ ), can reveal us the nature of the source.

Up to now the study of stellar populations in the inner Galaxy have mostly been restricted to low extinction fields, such as Baade's windows (see e.g. Rich 1988, Terndrup 1988). Recently, however, Ramírez et al. (2000b, hereafter referred to as RSFD) studied the $\mathrm{M}$ giant population in the inner Bulge using $K$-band spectra and determined a number of metallicities (see also Frogel et al. 1999; Ramírez et al. 2000a). Wood et al. (1998), Blommaert et al. (1998) and Ortiz et al. (2002) studied $\mathrm{OH} / \mathrm{IR}$ stars which are known to be the most extreme masslosing stars around the Galactic Center while Glass et al. (2001) performed a monitoring program of large-amplitude variables in the Galactic Centre.

In this work, we perform a spectroscopic follow-up study ( $H$ and $K$-bands) of 107 ISOGAL sources with IR excess at 7 and $15 \mu \mathrm{m}$ in the Inner Galactic Bulge to study their nature, mainly to identify young stellar objects and to establish criteria to distinguish between different classes of objects. We discuss the possibility of separating the different stellar populations such as AGB stars, $M$ giants, supergiants and young stellar objects in highly obscured regions $\left(A_{V} \sim 20-30 \mathrm{mag}\right)$, combining near-IR spectroscopy, near and mid-IR photometry and interstellar extinction data further. We discuss the massloss of AGB stars as well as of supergiants and red giant stars. Using the spectral features of $\mathrm{CO}, \mathrm{NaI}$ and $\mathrm{CaI}$, we estimate the metallicity and make a comparison to the solar neighbourhood sample of Lançon \& Wood (2000, hereafter referred to as LW). The derived metallicity distribution will be compared to the dissipative collapse model of Molla et al. (2000).

\section{Observations and data reduction}

\subsection{Near-IR observations}

The near-IR spectra were obtained between 16-19 July 2000 with the NTT telescope at ESO, La Silla, Chile, using the red grism of the SOFI spectrograph. The spectra were taken under photometric conditions through a $1^{\prime \prime}$ slit providing a resolving power of $R \sim 1000$, and were recorded on a Hawai HgCdTe $1024 \times 1024$ array. Before each spectrum, an image in the $K$ band was taken in order to check the identification of the source.

For optimal sky subtraction, "ABBA" observing sequences were used. The star was moved $10^{\prime \prime}$ along the slit between the A and $\mathrm{B}$ exposures. The exposure time was $30 \mathrm{~s}$ in each position, repeated 2-10 times, depending on the brightness of the object.

B, A, late F and early G spectrophotometric standard stars were observed during the night (typically 6-8 stars per night) to correct for telluric absorption features.

\subsection{Data reduction}

The data were reduced using MIDAS, the standard ESO reduction package. After removal of cosmic ray events, subtraction of the bias level and the dark, all frames were divided by a normalized flat field. The traces of stars at the two positions along the slit were used to subtract the sky. After extraction and co-adding of the spectra, a wavelength calibration was performed using the Xe-Ne lamp which gives an accuracy better than $2 \AA$. The spectra were rebinned to a linear scale, to obtain a dispersion of $\sim 10 \AA /$ pixel and a resulting wavelength range from $1.5 \mu \mathrm{m}$ to $2.5 \mu \mathrm{m}$.

We used B, A, F and G standard stars to correct for the instrumental and atmospheric transmissions. For each of the standard stars, a suitable model spectrum from Kurucz (1993) was selected. Significant stellar absorption features were removed by interpolation both in the models and the standard star observations $(\mathrm{Br} \gamma$ in $\mathrm{B}, \mathrm{A}$ stars; the strongest metal lines in $\mathrm{F}$ and $\mathrm{G}$ stars). The model spectra were then divided into the standard star spectra to provide the combined instrumental and atmospheric response. The choice of the standard used for the correction of a particular program star depended on proximity in time and airmass. We did not apply a second order correction for airmass, as the residual effects of the telluric bands on the features we were interested in are small compared to intrinsic variations. For a more detailed discussion on spectroscopic data reduction in general, we refer the reader to LW. The final spectra are normalized at $2.28 \mu \mathrm{m}$ and are dereddened (see Sect. 3.1) using the extinction values of Schultheis et al. (1999). They are displayed in Appendix B. Their classification is discussed in Sect. 4. The spectra are available electronically at CDS together with the corresponding finding charts.

\subsection{Equivalent widths}

Prominent spectral features in our data are the NaI, CaI and $\mathrm{CO}(2,0)$ bands which have also been discussed by Ramírez et al. (1997, hereafter referred to as RDF), and the $\operatorname{CO}(6,3)$ 
Table 1. Definition of bandpasses for continuum and features.

\begin{tabular}{lcl}
\hline \hline Feature & Band passes $(\mu \mathrm{m})$ & Reference \\
\hline NaI feature & $2.204-2.211$ & RDF \\
NaI continuum \#1 & $2.191-2.197$ & RDF \\
NaI continuum \#2 & $2.213-2.217$ & RDF \\
CaI feature & $2.258-2.269$ & RDF \\
$\mathrm{CaI}$ continumm \#1 & $2.245-2.256$ & RDF \\
$\mathrm{CaI}$ continuum \#2 & $2.270-2.272$ & RDF \\
${ }^{12} \mathrm{CO}(2,0)$ bandhead & $2.289-2.302$ & LW \\
${ }^{12} \mathrm{CO}(2,0)$ continuum\#1 & $2.242-2.258$ & LW \\
${ }^{12} \mathrm{CO}(2,0)$ continuum\#2 & $2.284-2.291$ & LW \\
$\mathrm{H}_{2} \mathrm{O}$ continuum & $1.629-1.720$ & \\
$\mathrm{H}_{2} \mathrm{O}$ absorption wing 1 & $1.515-1.525$ & \\
$\mathrm{H}_{2} \mathrm{O}$ absorption wing 2 & $1.770-1.780$ & \\
\hline
\end{tabular}

and SiI lines (see Origlia et al. 1993 for details). In addition, the $\mathrm{OH}$ radical has many groups of prominent lines scattered over the whole $\mathrm{H}$ window (Origlia et al. 1993, LW)

In our analysis we will use the equivalent widths of the ${ }^{12} \mathrm{CO}(2,0)$ bands at $2.3 \mu \mathrm{m}(E W(\mathrm{CO}))$ and the equivalent widths of the $\mathrm{NaI}(E W(\mathrm{Na}))$ and $\mathrm{CaI}(E W(\mathrm{Ca}))$ lines. Additionally, the water absorption $\left(E W\left(\mathrm{H}_{2} \mathrm{O}\right)\right)$ at $\sim 1.6 \mu \mathrm{m}$ has been measured. The adopted index measures the curvature of the spectrum in the $\mathrm{H}$ window due to the wings of the water bands centered at 1.4 and $1.9 \mu \mathrm{m}$. The index compares the flux in a central passband that is only weakly contaminated by $\mathrm{H}_{2} \mathrm{O}$, to the fluxes in passbands on either sides. For consistency with the units of the other feature measurements, its formal expression is that of an equivalent width (but it takes negative values when $\mathrm{H}_{2} \mathrm{O}$ absorption is present). The measured features are identified in Fig. 2. The exact bandpasses for all measurements are provided in Table 1.

\subsection{The ISOGAL catalogue}

The final product of the ISOGAL catalogues (ISOGAL PSC) at present gives magnitudes, $I, J, K_{\mathrm{S}}$, [7], [15], at five wavelengths $(0.8,1.25,2.15,7$ and $15 \mu \mathrm{m})$ with DENIS providing $I, J, K_{\mathrm{S}}$ associations when available. We refer for a detailed description of data reduction and the cross-identification method to Schuller et al. (2003). Note that here and elsewhere we use $[\lambda]$ to denote the ISOGAL magnitude at wavelength $\lambda \mu \mathrm{m}$ ([7], [15])

\section{Sample}

Figure 1 shows the location of the ISOGAL sources observed with SOFI superimposed on the extinction map of Schultheis et al. (1999). The sources were selected from the ISOGAL fields located at $l=-0.27^{\circ}, b=-0.03^{\circ}$ (hereafter referred to as field A), $l=-0.62^{\circ}, b=-0.03^{\circ}$ (field B), $l=-0.90^{\circ}$, $b=-0.03^{\circ}$ (field C), $l=-1.21^{\circ}, b=-0.03^{\circ}$ (field D), $l=-0.44^{\circ}, b=-0.18^{\circ}$ (field E), $l=-1.12^{\circ}, b=+0.30^{\circ}$ (field F), $l=-1.12^{\circ}, b=-0.33^{\circ}$ (field $\mathrm{G}$ ), $l=-5.76^{\circ}$, $b=+0.17^{\circ}$ (field $\mathrm{H}$ ). Precise details of the fields can be found in the Explanatory Supplement of the ISOGAL catalog (Schuller et al. 2003). We selected sources with [7]-[15] $>1.4$ and [15] $<8.0$ (except for 4 cases, see Fig. 5). These colour criteria were adopted to favour the detection of dusty young objects with mid infrared excesses close to the galactic centre, but they are also satisfied by a number of evolved stars, as discussed in Sect. 4. We further restricted the sample to sources brighter than $\sim 11 \mathrm{mag}$ in $K_{\mathrm{S}}$ in order to avoid spurious associations (Schuller et al. 2003). Such a value is slightly brighter than the $K_{\mathrm{S}}$ completeness limit of DENIS in these regions (see also Schultheis \& Glass 2001). From the 1130 ISOGAL targets obeying the above criteria in these fields, the 107 sources actually observed with SOFI were selected approximately at random, with the majority of the sources (65) in field A.

After the observations, we cross-identified our sample again with the latest version of the ISOGAL Point Source Catalogue (Version 1) described in Schuller et al. (2003) in order to get the final DENIS and ISO photometry. We have kept only sources which have a good quality association (quality flags 4 and 5; see Schuller et al. 2003 for details). 5\% of our sample now show a more doubtful association between ISOGAL and DENIS (quality flag < 3) and have been dropped. Table A.1 lists the coordinates and the corresponding ISOGAL and DENIS magnitudes as well as the measured equivalent widths and the type of each source.

As a comparison sample, we used the low resolution $(R \sim 1300) K$-band spectra of M giants of RSFD which are located at $\left(l=0, b=-1.3,-1.8,-2.3,-2.8^{\circ}\right)$ and at $\left(b=-1.3^{\circ}\right.$, $\left.l=1.0,2.0,3.0,4.0^{\circ}\right)$ and the solar neighbourhood sample of oxygen-rich stars ( $\mathrm{M}$ giants, semi-regular Variables and Mira Variables) of LW.

\subsection{Interstellar reddening}

The high interstellar extinction in the Galactic Bulge hampers the study of the stellar populations in the inner parts of our Galaxy. As in most parts of the Galactic Bulge, interstellar absorption is not homogeneous but occurs in clumps (Glass et al. 1987). Catchpole et al. (1990) mapped the interstellar extinction around the Galactic Centre $\left(\sim 2 \mathrm{deg}^{2}\right)$ using the red giant branch of 47Tuc as a reference while Frogel et al. (1999) determined the interstellar reddening for a few fields in the inner Galactic Bulge using the red giant branch of Baade's window. Schultheis et al. (1999) constructed a high resolution map of the whole inner part using DENIS data together with isochrones calculated for the RGB and AGB phases. We used their extinction table to deredden our objects (see Table A.1) according to the interstellar extinction law of Glass (1999) with $A_{V}: A_{J}: A_{K}=1: 0.256: 0.089$. For 7 and $15 \mu \mathrm{m}$ photometry we used $A_{7} / A_{V}=0.020$ and $A_{15} / A_{V}=0.025$ (Hennebelle et al. 2001). However, the extinction curve particularly at 7 and $15 \mu \mathrm{m}$ is rather uncertain. One has also to keep in mind that such extinction values correspond to the peak value of $A_{V}$ on the line of sight; but the actual value for each individual star may differ by several magnitudes. In addition, the extinction values in the very high extincted regions $\left(A_{V}>25\right)$ are only lower limits. Nevertheless there seems to be on average 


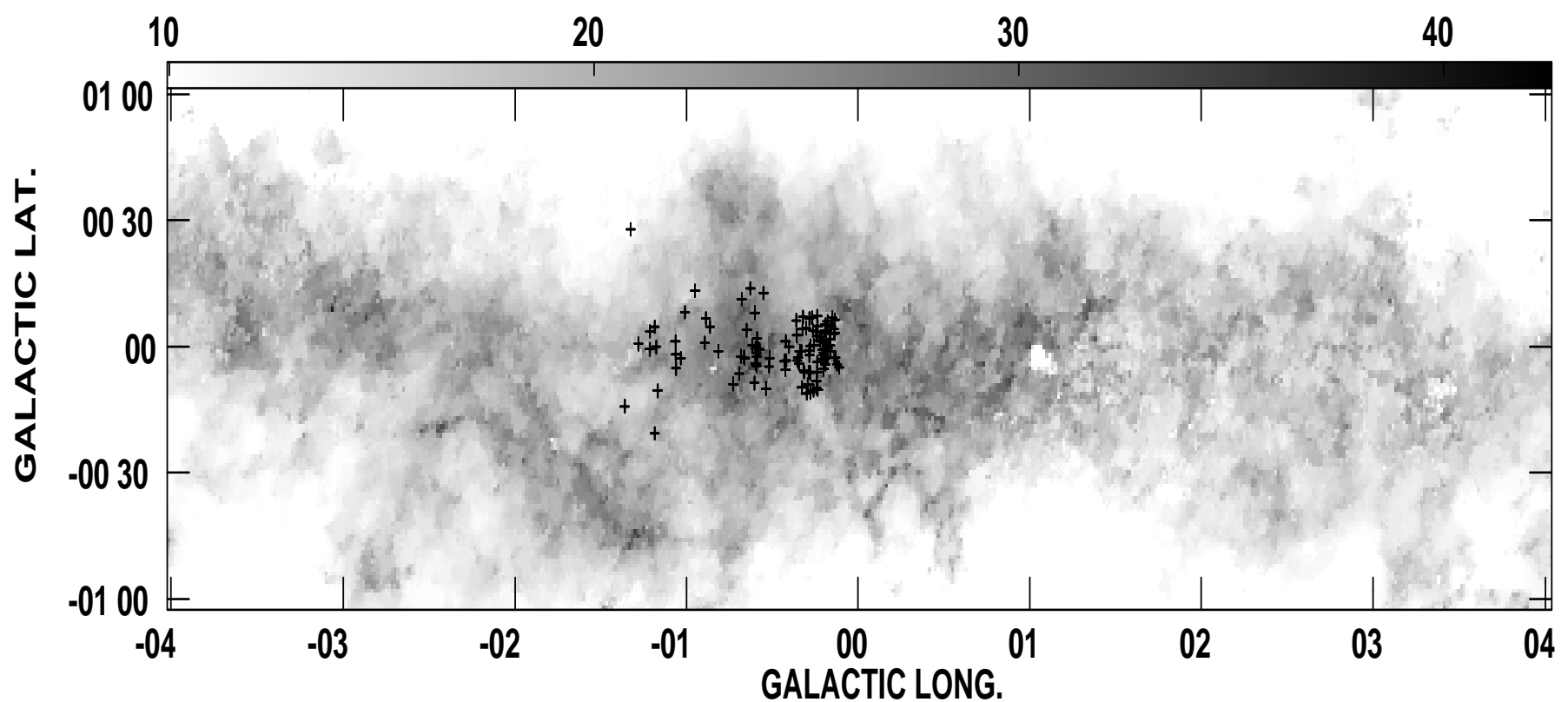

Fig. 1. Observed near-IR spectra (indicated by plus signs) superimposed on the extinction map by Schultheis et al. (1999). The greyscale on the top shows the range of $A_{V}$. Galactic longitude and latitude are given in units of degrees. Note the high interstellar extinction with $A_{V}>20$ mag. The comparison fields of RSFD are located at $\left(l=0, b=-1.3,-1.8,-2.3,-2.8^{\circ}\right)$ and $\left(b=-1.3^{\circ}, l=1.0,2.0,3.0,4.0^{\circ}\right)$.

a reasonable agreement with the $A_{V}$ values derived by Wood et al. (1998) for OH/IR stars as discussed by Ortiz et al. (2002).

\subsection{Bolometric magnitudes}

Bolometric magnitudes for our ISOGAL objects were obtained by using the multi-band bolometric correction for AGB stars (Loup et al. 2003). It employs multi-band photometry with passbands as accurate as possible, taking into account a mean atmospheric absorption at the site for ground-based observations. The bolometric corrections are derived numerically using 69 models for O-rich stars (see Loup et al. 2003 for a detailed description). The models are based on Groenewegen's radiative transfer code, spanning a large range of dust opacities. The advantage of multi-band bolometric corrections is that the determination of $M_{\text {bol }}$ is more accurate than with traditional oneband bolometric corrections. We used for the input parameter the DENIS $J$ and $K_{\mathrm{S}}$ counterparts, the $L W 2$ and $L W 3$ magnitudes of our objects and the $A_{V}$ values of Schultheis et al. (1999). The main errors in the bolometric corrections result from the interstellar extinction values giving an error in $m_{\mathrm{bol}}$ of at least $\sim 0.2-0.3$ mag and the intrinsic depth of the "Bulge" on the line of sight (Alard et al. 2001, OOS). Variability of the stars will introduce additional errors in the determination of $M_{\mathrm{bol}}$ because of non-simultaneity of the photometry. In Table 2 we list the derived $M_{\mathrm{bol}}$ using a distance modulus of $\Delta m=14.5$ (or a distance of $\sim 8 \mathrm{kpc}$ ).

\subsection{Cross-identification with SIMBAD}

All the objects have been searched for in the SIMBAD data base using a search radius of $3^{\prime \prime}$. As a result, fifteen objects have been identified as $\mathrm{OH} / \mathrm{IR}$ stars from the sample of Wood et al. (1998, see also Ortiz et al. 2002), and twelve as Long

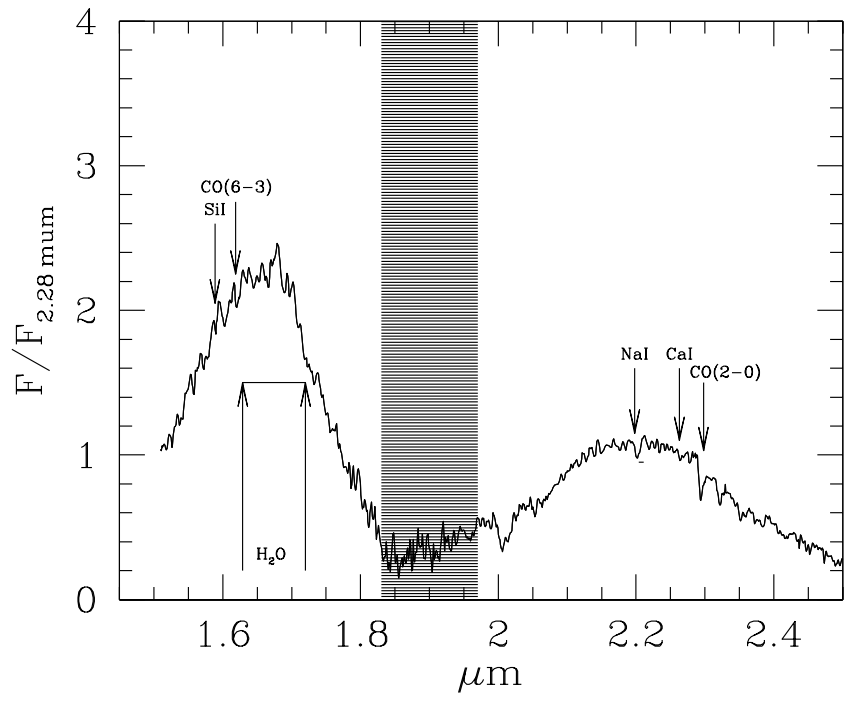

Fig. 2. Spectrum of an AGB star superimposed by the most prominent features of SiI, $\mathrm{CO}(6-3)$, NaI, $\mathrm{CaI}, \mathrm{CO}(2-0)$ and the $\mathrm{H}_{2} \mathrm{O}$ band. The shaded area indicates the region of very strong telluric absorption.

Period variables from Glass et al. (2001). These subsamples are shown, respectively, in Figs. B.3 and B.4. Four objects (A33, A40, D6, D7) are known radio sources with IRAS fluxes characteristic of young stellar objects(see also Sect. 4.3).

\section{Classification}

Figure 3 shows the bolometric magnitudes vs. the [7]-[15] colour ([7] and [15] denote the magnitude at 7 and $15 \mu \mathrm{m}$ ), which is nearly insensitive to interstellar extinction. Indicated also are the known OH/IR stars and LPVs in our sample (see Sect. 4.2). It is obvious from Fig. 3 that photometric 


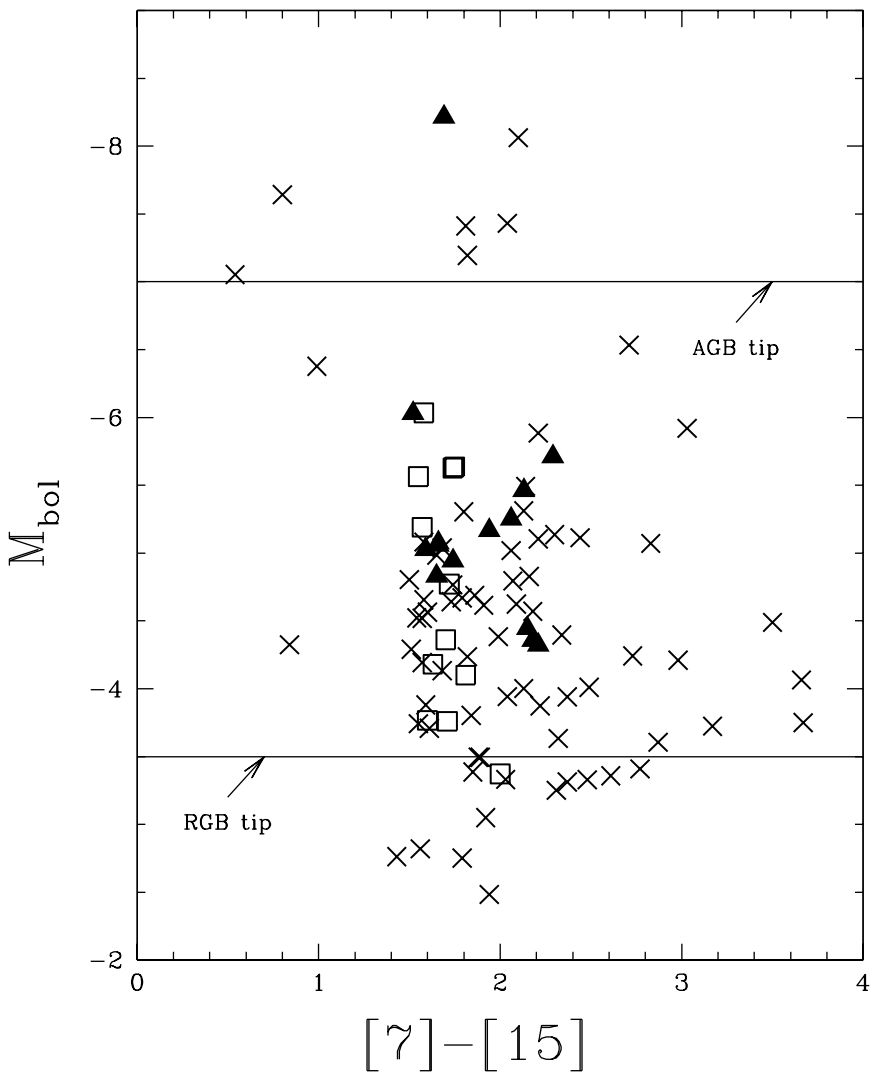

Fig. 3. $M_{\text {bol }}$ vs. [7]-[15] diagram for the ISOGAL+SOFI sample. Known OH/IR stars (Wood et al. 1998) are indicated by filled triangles, LPVs (Glass et al. 2001) by open squares, while the remaining ISOGAL objects of our sample are shown by crosses. The two lines indicate the tip of the RGB and AGB.

information alone is not sufficient to separate the different categories: additional spectroscopic observations are necessary.

\subsection{Supergiants}

As shown in Fig. 4, we find six very luminous objects (A17, B14, B15, B19, E5, E13), besides one OH/IR star, with $M_{\text {bol }} \lesssim-7.0$ (or $\gtrsim 50000 L_{\odot}$ ), assuming a distance of $8 \mathrm{kpc}$ to the Galactic Center), which is approximately the AGB tip luminosity (Vassiliadis \& Wood 1993). They show very strong CO bands at $2.3 \mu \mathrm{m}$ with equivalent widths $>20 \AA$ (see Sect. 2.3 for the band passes). In addition, four of them (A17, B14, B15, B19) do not show any significant $\mathrm{H}_{2} \mathrm{O}$ absorption $\left(E W\left(\mathrm{H}_{2} \mathrm{O}\right)<100 \AA\right)$ which is an indication of supergiants (Bessell et al. 1991; Lançon \& Rocca-Volmerange 1992). The objects E5 and E13 show very blue colours in $K_{0}-$ [15] and [7]-[15] and could be late K or early M-type supergiant candidates. Schuller (2003) found from a systematic search of very luminous ISOGAL sources $\left(M_{\mathrm{bol}}<-6\right)$ several blue supergiant candidates with $K_{0}-[15]<1.0$.

In the [15] vs. [7]-[15] diagram (see Fig. 4a), the supergiants (stars) are rather luminous at $15 \mu \mathrm{m}$ and show rather blue $K_{0}-[15]$ colours compared to the AGB variables (see Fig. 4b).

\subsection{Long Period variables}

The majority of our sources we find to be Long-Period Variables (LPVs) of the AGB, mainly based on their very deep water absorption features (LW and Fig. 3a). Strong $\mathrm{H}_{2} \mathrm{O}$ bands are associated with large period variability and depend on the atmospheric structure of the star (see Bessell et al. 1996). Their luminosities are in the range $-4<M_{\text {bol }}<-7$ and their strong $\mathrm{CO}$ bands, characteristic of stars on the AGB, also imply that they are likely to be Miras and semi-regular variables (SRVs). Figure 6 shows a comparison between the water bands and the CO bands of the ISOGAL sample and a solar neighbourhood sample of semi-regular variables and Miras (LW). The samples agree surprisingly well within the broad range of the band strength values of both bands. We identify the ISOGAL sources having strong $\mathrm{H}_{2} \mathrm{O}$ absorption as AGB variables (semi-regular variables or Miras). Greene \& Lada (1996) and Gómez \& Mardones (2003) showed that YSOs of class III can show rather strong $\mathrm{H}_{2} \mathrm{O}$ and $\mathrm{CO}$ bands too. However, they have rather low luminosities with $M_{\text {bol }}>2$ (see Gómez \& Mardones 2003), much too faint to be on the AGB $\left(M_{\mathrm{bol}}<-3.5\right)$ Therefore, only additional information about the luminosity give us the real confirmation that our spectra of luminous stars with strong water absorption are indeed AGB star candidates (see Fig. 4).

In Fig. 4a one can see, except for supergiants and the other very bright sources $\left(M_{\text {bol }} \lesssim-6.0\right)$, some correlation between the strength of the water bands and the bolometric magnitude in the sense that more luminous objects show deeper water absorption. The [15] vs. $K_{0}-$ [15] diagram (Fig. 5) shows that the LPVs have a wide $K_{0}-[15]$ colour range $\left(0<K_{0}-[15]<7\right)$ indicating a large range of mass-loss rates (see discussion below).

Twelve LPVs from our sample and five OH/IR stars (see Table A.1) were observed by Glass et al. (2001) and we have therefore additional information about their periods and their $K$ amplitudes. The period range is rather narrow, starting from $\sim 400^{\mathrm{d}}$ up to $800^{\mathrm{d}}$. The sources within the sample follow a period-luminosity relation but we do not find any relation between the $\mathrm{CO}$ or water band strengths and the amplitude.

\subsection{Young stellar objects}

A33, A40, D6 and D7 are already known to be young stellar objects. They have been detected by IRAS with very red colours and also show radio emission. They have more-or-less featureless spectra (see Fig. B.1) with no $\mathrm{CO}$ absorption at $2.3 \mu \mathrm{m}$ but in some cases possess hydrogen absorption lines (Brackett series). The objects B23, B27, B35, B37, C19 are newly found young stellar objects showing the same spectral features as the four known ones.

Greene \& Lada (1996) presented the first systematic spectroscopic survey of YSOs, comprising a sample from the Ophiucus molecular cloud. They found that the strengths of atomic and $\mathrm{CO}$ absorption features are closely related to the evolutionary state. The line strengths decrease from the Class III phase through Class II to the self-embedded Class I, where the absorption features are absent. All nine YSOs of our sample show more-or-less featureless spectra with no $\mathrm{CO}$ 

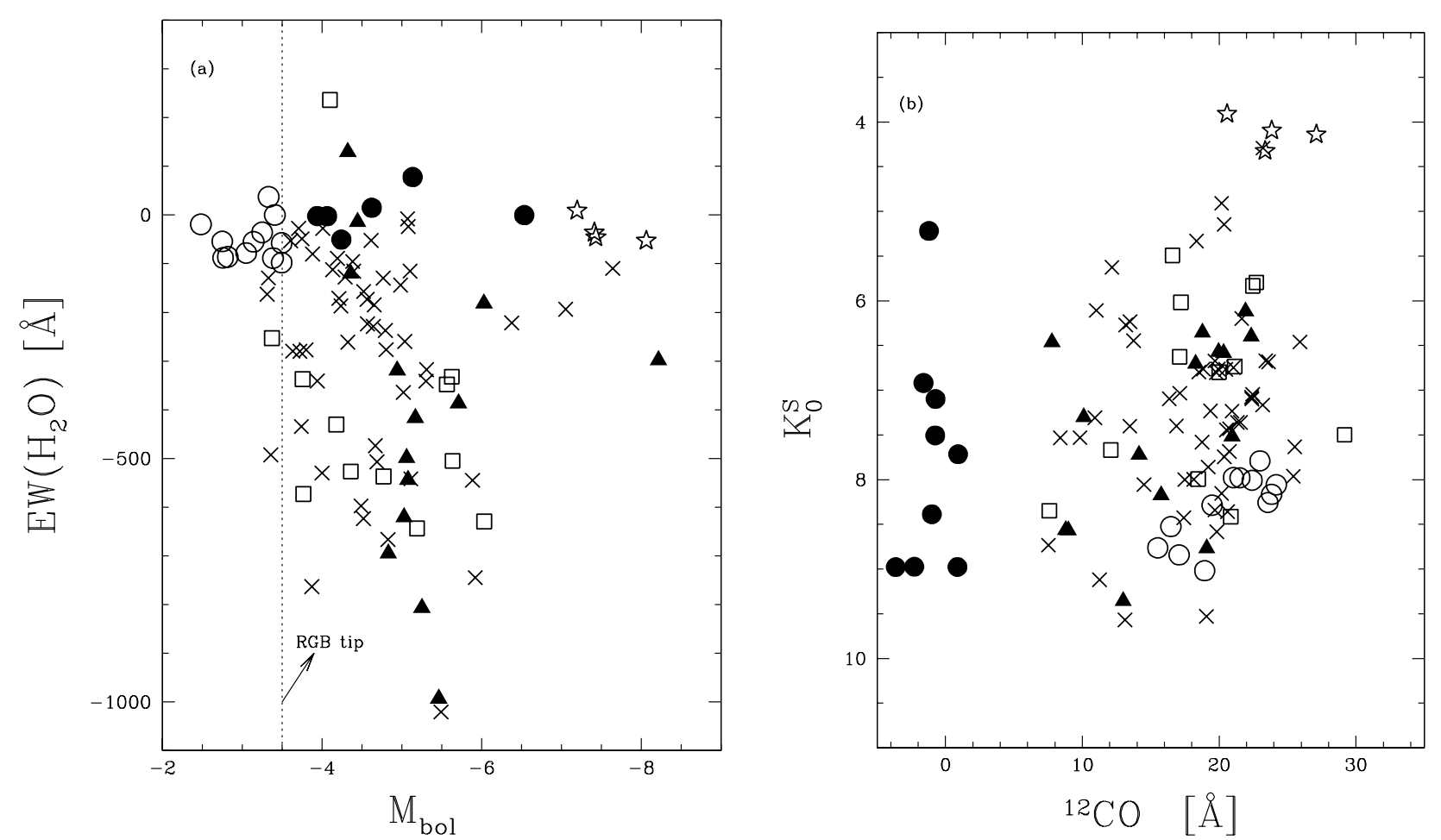

Fig. 4. Equivalent width of $\mathrm{H}_{2} \mathrm{O}$ vs. $M_{\text {bol }}$ a) and $K_{0}$ vs. equivalent width of CO b). YSOs are indicated by filled circles, OH/IR stars (Wood et al. 1998) by filled triangles, LPVs (Glass et al. 2001) by open squares, candidates of red giants by open circles, supergiant candidates by stars and AGB Variables by crosses. The dotted line indicated the tip of the RGB. The two AGB stars with $M_{\text {bol }} \leq-7$ are probably blue supergiant candidates (see text).

absorption lines and no $\mathrm{Br} \gamma$ emission. Thus, we associate them with young stellar objects of Class I.

However, Fig. 5 shows that YSOs cannot be separated unambiguously from other stellar populations by using ISOGAL colours alone. Felli et al. (2000) give a criterion for selecting YSO candidates (see Fig. 4) in the ISOGAL [15] vs. [7]-[15] diagram and Felli et al. (2002) present a catalog of YSO candidates with [15] $<4.5$ and [7]-[15] $>1.8$. The criteria defined by Felli et al. (2002) are satisfied by seven of the nine identified YSOs in this region (see Fig. 4a). However, as shown in Fig. 5, there are also $32 \mathrm{AGB}$ variables, eight $\mathrm{OH} / \mathrm{IR}$ stars and four supergiants in the same region. Felli et al. (2002) point out also that $[7]-[15] \geq 2.5$ is a more conservative criterion for identifying YSOs. Six of the YSOs meet this criterion but it is still satisfied by seven objects which are probably AGB stars. Thus, an unambiguous separation between YSOs and evolved stars can only be made by using additional spectroscopic information.

\section{4. $M$ giants}

In contrast to the typical LPV, M giants show nearly no water vapor bands. Using an additional luminosity criterion, namely $M_{\text {bol }}$ fainter than -3.5 (see Fig. 3), which is approximately the tip of the RGB (see Tiede et al. 1996; Omont et al. 1999), we find 12 objects. Figure 5 shows that indeed the M giant candidates populate the lower end in both the [15] vs. [7]-[15] and [15] vs. $K_{0}-$ [15] diagram. However, as seen in Fig. 6, the separation between $\mathrm{M}$ giants and variable AGB stars in the CO vs. $\mathrm{H}_{2} \mathrm{O}$ plane is ambiguous. Some of the stars classified as giants might be AGB stars that happen to be caught at a phase of pulsation where $\mathrm{H}_{2} \mathrm{O}$ is not very strong (see LW). Additional multi-epoch observations are necessary, especially because it has recently been shown that most M giants with late spectral types are variable (Alcock et al. 2000; Glass \& Schultheis 2002).

\section{Discussion}

Figure 5 shows that near and mid-IR photometry alone are not sufficient for distinguishing the different stellar populations; additional near-IR spectroscopy is necessary. Using the $M_{\text {bol }}$ vs. $\mathrm{H}_{2} \mathrm{O}$ and the ${ }^{12} \mathrm{CO}$ vs. $K_{0}$ diagram (see Fig. 4 ) we can identify supergiants, AGB Variables, red giants and young stellar objects. In addition, mid-IR data at 7 and $15 \mu \mathrm{m}$ enables us to estimate mass-loss rates of the stellar population while the equivalent widths of the $\mathrm{CO}$ bandhead, the $\mathrm{NaI}$ doublet and the $\mathrm{CaI}$ triplet give us estimates of the metallicity.

\subsection{Mass loss}

One of the most promising tools for determining mass-loss rates is the combination of near-IR and mid-IR colour such as the IRAS $K_{0}-[12]$ or the ISOGAL $K_{0}-[15]$ colours (see e.g. Whitelock et al. 1994; Habing 1996; Le Bertre \& Winters 1998; Omont et al. 1999; Jeong et al. 2002, OOS etc.). We will use the most recent colour-mass loss relation for oxygen-rich AGB stars by Jeong et al. (2002) which is based on a consistent 

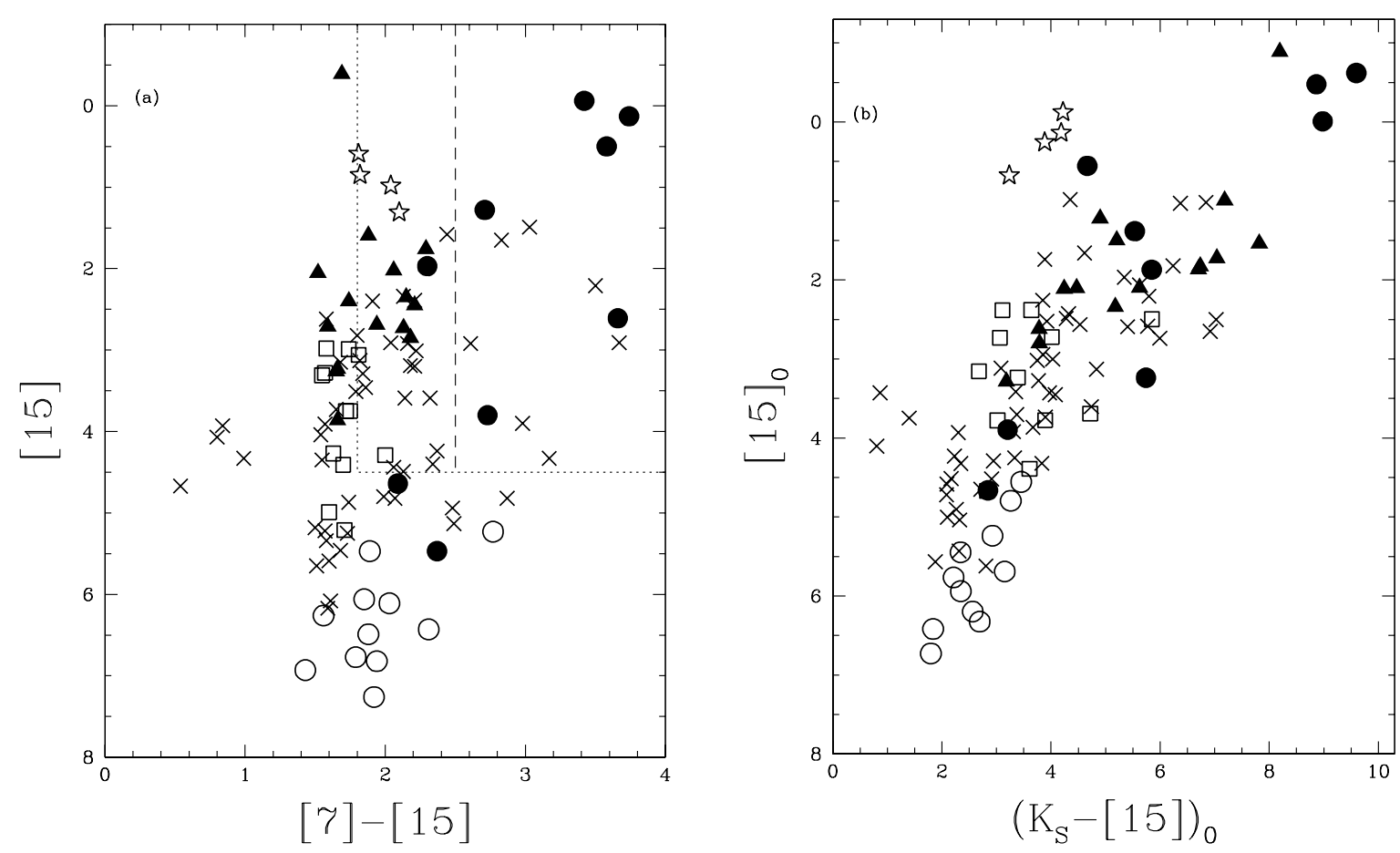

Fig. 5. [15] vs. [7]-[15] diagram (left panel) and [15] vs. $K_{0}-$ [15] diagram (right panel). Symbols are the same as in Fig. 4. The dotted line shows the region searched by Felli et al. (2000) for YSO candidates (see text). The long-dashed line indicates a more conservative criterion for identifying YSOs (Felli et al. 2002).

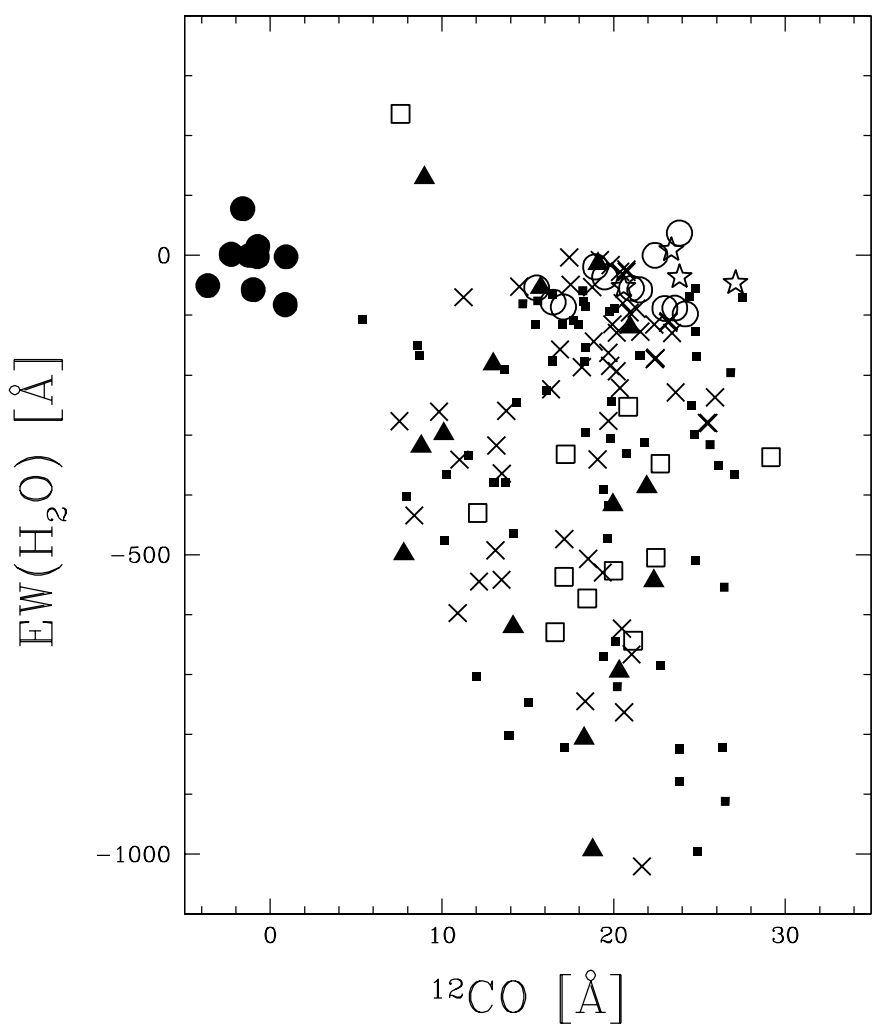

Fig. 6. Equivalent width of $\mathrm{H}_{2} \mathrm{O}$ vs. $\mathrm{CO}$, using the sample of oxygenrich LPVs of LW for comparison. YSOs are indicated by filled circles, $\mathrm{OH} / \mathrm{IR}$ stars by filled triangles, LPVs by open squares, candidates of giants by open circles, supergiant candidates by stars and AGB variables by crosses. Filled squares indicate the comparison sample of semi-regular variables and Miras of LW. time dependent treatment of hydrodynamics, thermodynamics, equilibrium chemistry and dust formation. They give an explicit relation between $\dot{M}$ and the $(K-[15])_{0}$ colour.

$\log \dot{M}=-6.83 /(K-[15])_{0}-3.78 \quad\left[M_{\odot} / \mathrm{yr}\right]$.

One has to be aware that using this relation between $\dot{M}$ and $K_{0}-$ [15] for our sample includes uncertainties in the determination of the mass-loss rate arising from the following causes: (1) Our de-reddend $(K-[15])_{0}$ colours are strongly affected by the uncertainty in the determination of interstellar extinction, in particularly in these highly extincted regions (see Schultheis et al. 1999). This could lead easily to errors in $\dot{M}$ of a factor of $2-3$; (2) The relation was derived for oxygen-rich AGB stars in the solar neighbourhood. Habing et al. (1994) argued that metallicity affects the dust to gas ratio and the outflow velocity from evolved stars which is directly related to the massloss and that, therefore, the color $-\dot{M}$ relation might differ in different galactic environments such as between the Galactic Bulge and the Magellanic Clouds; (3) It is important to emphasize that the $K_{\mathrm{S}}$ magnitudes of DENIS are single epoch measurements. The average $K$ amplitude of our sources associated with known LPVs (Glass et al. 2001) is $\sim 1.0 \mathrm{mag}$, which gives a factor of $\sim 2-5$ uncertainty in the determination of $\dot{M}$ for $-7<\log \dot{M}<-5$. We want to emphasize that the colour mass-loss relation by Jeong et al. (2002) is calculated for variable AGB stars. Its adaption to non-variable red giants and supergiants has to be questioned.

From this relation, the $\mathrm{OH} / \mathrm{IR}$ stars in our sample cover mass-loss rates ranging from $-6.0<\log \dot{M}<-4.5$ while LPVs range between $-6.5<\log \dot{M}<-5$. Considering the uncertainties (see above) these values lie within the ranges 


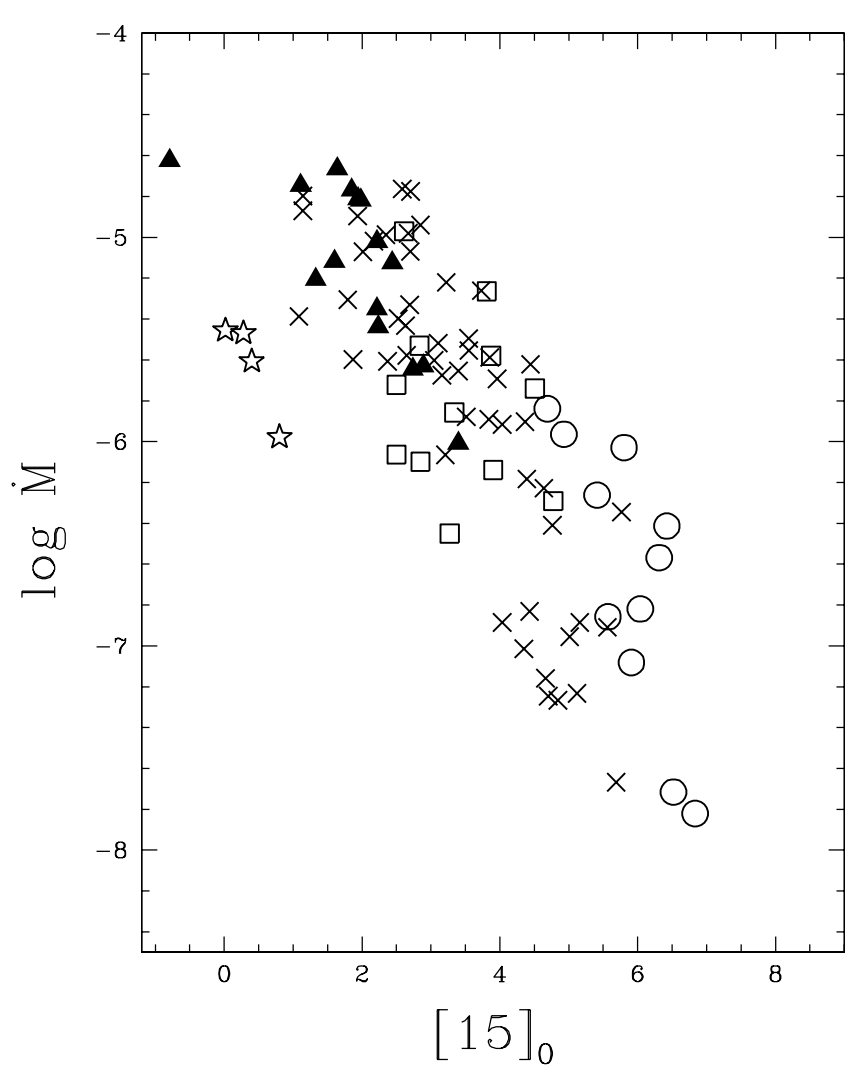

Fig. 7. Mass-loss rate vs. $15 \mu \mathrm{m}$ magnitude vs. for the present sample. $\mathrm{OH} / \mathrm{IR}$ stars are indicated by filled triangles, LPVs by open squares, red giant candidates by open circles and supergiants by stars.

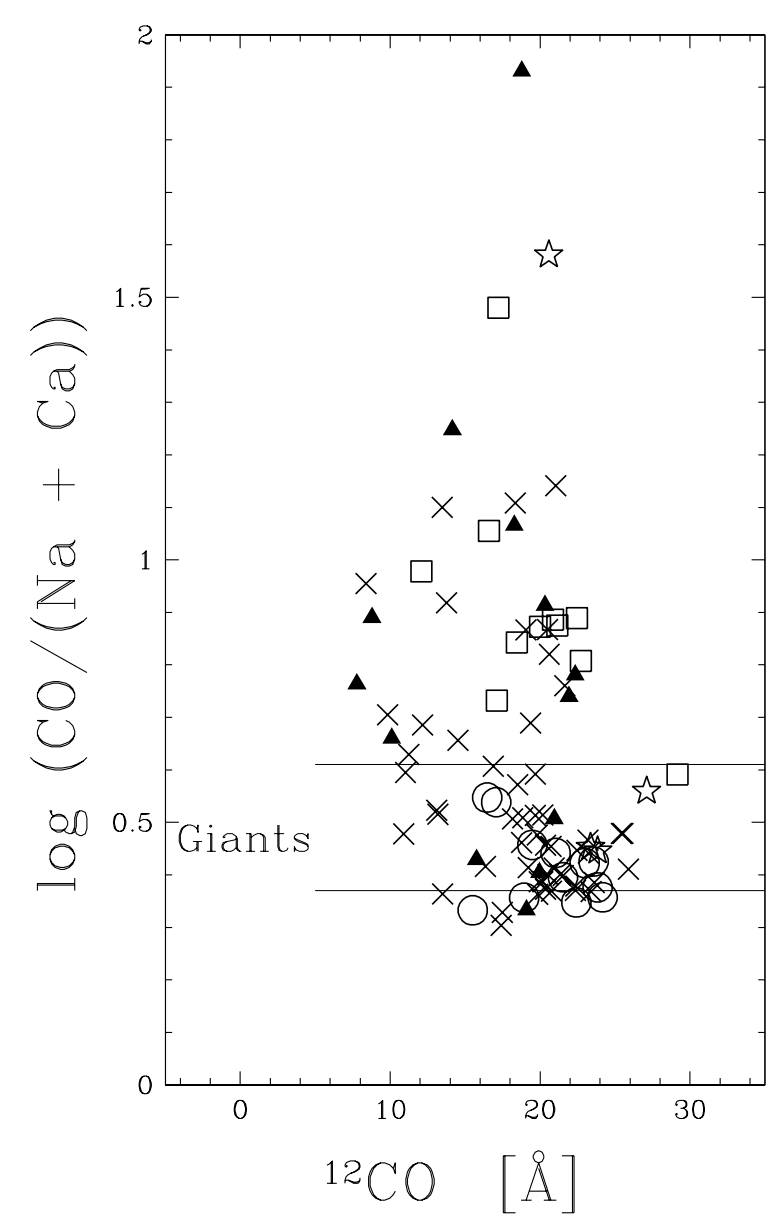

Fig. 8. The luminosity indicator of Ramírez et al. (1997) vs. CO band strengths. The two straight lines show the location of the $\mathrm{M}$ giants of Ramírez et al. (1997). The symbols are the same as in Fig. 3.

\subsection{Spectral determination of luminosity class}

Do spectra in the $H$ and $K$ bands offer the possibility of determining the luminosity class? Ramírez et al. (1997) used the quantity $\log [E W(\mathrm{CO}) /(E W(\mathrm{Na})+E W(\mathrm{Ca}))]$ to distinguish between giants and dwarfs over the effective temperature range between 3400 and $4600 \mathrm{~K}$ (see their Fig. 11). They argued that this quantity might be a powerful luminosity diagnostic. Figure 8 shows that the $M$ giants of RSFD (indicated by the two straight lines) and our sample agree quite well. For comparison, we calculated also the values for the LW sample. We find that there is no clear separation between supergiants and $\mathrm{M}$ giants or LPVs. However, as seen in Fig. 8, the dispersion in $\log [E W(\mathrm{CO}) /(E W(\mathrm{Na})+E W(\mathrm{Ca}))]$ is much narrower for $\mathrm{M}$ giants than for variable AGB stars. Pulsation is responsible for the extended atmospheres of LPVs (Feuchtinger et al. 1993) which is seen in the large dispersion of $\log [E W(\mathrm{CO}) /(E W(\mathrm{Na})+E W(\mathrm{Ca}))]$ of the LPVs and $\mathrm{OH} / \mathrm{IR}$ stars. We get similar results using the LW sample. While the quantity $\log [E W(\mathrm{CO}) /(E W(\mathrm{Na})+E W(\mathrm{Ca}))]$ is a good discriminator between dwarfs and giants, it can not be adopted to separate LPVs from supergiants and red giants. 


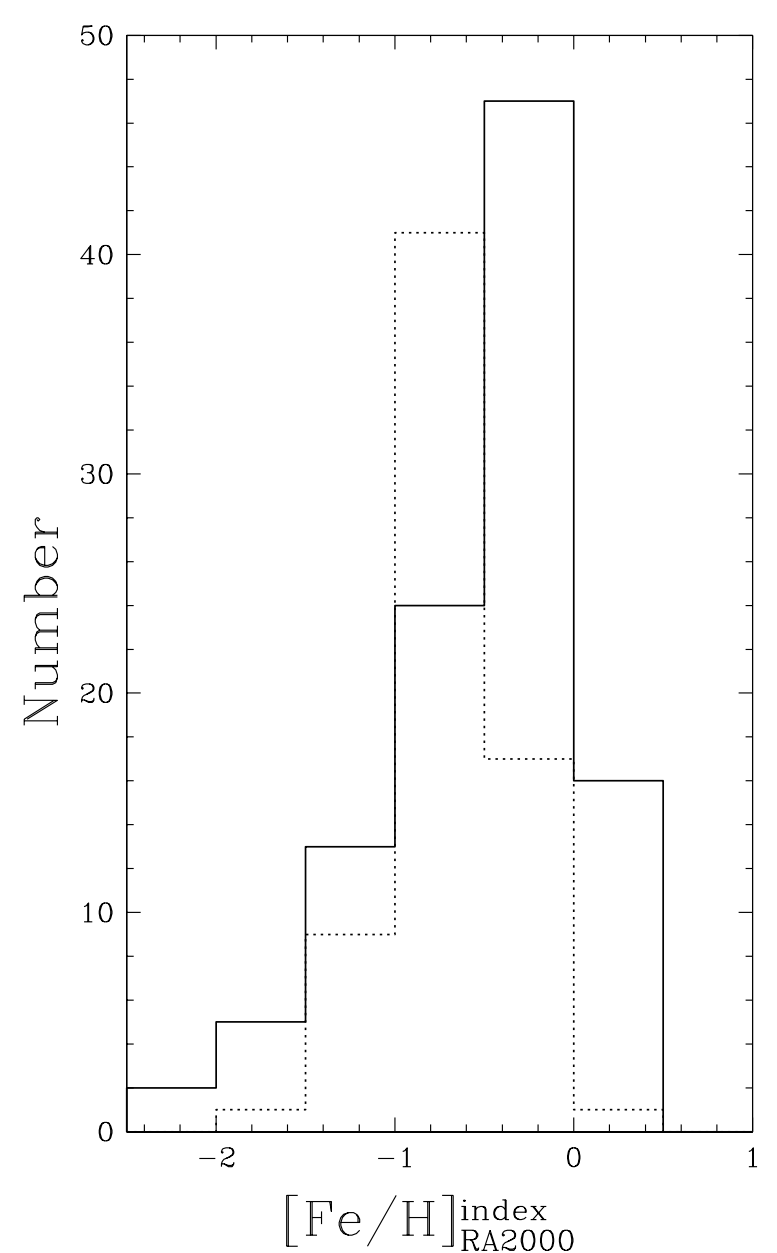

Fig. 9. Distribution of metallicity. The solid lines is our ISOGAL sample located in the inner Bulge; the dotted line is the solar neighbourhood sample of oxygen-rich AGB stars and M giants by LW.

\subsection{Metallicity}

Recently, RSFD and Frogel et al. (2001) have obtained a new metallicity scale for luminous red giants based on equivalent width measurements of the $\mathrm{CO}$ bandhead, the NaI doublet and the CaI triplet (see Table 1). Their calibration is based on giants in globular clusters for $-1.8<[\mathrm{Fe} / \mathrm{H}]<-0.1$. We have used the following relation (solution $1, \mathrm{RSFD}$ )

$$
\begin{aligned}
{[\mathrm{Fe} / \mathrm{H}]_{\mathrm{RSFD}}^{\text {index }}=} & -1.782+0.352 E W(\mathrm{Na})-0.0231 E W(\mathrm{Na})^{2} \\
& -0.0267 E W(\mathrm{Ca})+0.0129 E W(\mathrm{Ca})^{2} \\
& +0.0472 E W(\mathrm{CO})-0.00109 E W(\mathrm{CO})^{2}
\end{aligned}
$$

where $E W(\mathrm{Na}), E W(\mathrm{Ca})$ and $E W(\mathrm{CO})$ are the equivalent widths of NaI, $\mathrm{CaI}$ and ${ }^{12} \mathrm{CO}(2,0)$ (see Table 1). As described in RA97, the typical errors in the determination of $[\mathrm{Fe} / \mathrm{H}]$ are of the order of $\sim 0.1$ dex. RSFD provide a second expression for $[\mathrm{Fe} / \mathrm{H}]_{\mathrm{RSFD}}^{\mathrm{index}}$, that reduces the scatter in their data with correction terms based on $(J-K)_{0}$. As most of our program stars do not have measurable $J$ magnitudes due to heavy extinction, this second relation could not be used.

In addition, we determined this index from Eq. (2) for the sample of oxygen-rich stars (M giants, semi-regular variables and Miras) in the solar neighbourhood of LW using the same bandpasses. We used repeated observations of LW for semiregular and Mira variables, such as BD Hya, R Cha or KV Car. For the same star the metallicity index of RSFD can vary by $\sim 0.5$ dex showing that it does not actually measure metallicity in individual spectra of strongly variable stars.

Figure 9 shows the distribution of the RSFD metallicity index of our ISOGAL objects superimposed on the solar neighbourhood sample of LW. If we use only the non-varying static stars of LW and our sample of red giant candidates, the mean $[\mathrm{Fe} / \mathrm{H}]_{\mathrm{RSFD}}^{\mathrm{index}}$ does not change, although the dispersion gets smaller. This shows that, even if the metallicity index of RSFD is not strictly valid for single observations of a variable star, it can be used on average over a whole pulsation cycle. This is important for stellar population studies, as our results show that very time consuming multi-epoch observations of LPVs are not necessary in order to obtain average metallicities. We will only use average values in the remaining discussion.

The mean $[\mathrm{Fe} / \mathrm{H}]_{\mathrm{RSFD}}^{\text {index }}$ is -0.25 dex, with a dispersion of \pm 0.40 dex, which is in agreement with the values obtained for the static sample of RSFD. Their mean $[\mathrm{Fe} / \mathrm{H}]_{\mathrm{RSFD}}^{\text {index }}$ is -0.21 dex with a dispersion of \pm 0.30 dex. This suggests that the ISOGAL sample and the sample of RSFD have similar metallicities despite their spatial separation.

Superimposed is the solar neighbourhood sample of LW with an average $[\mathrm{Fe} / \mathrm{H}]_{\mathrm{RSFD}}^{\text {index }}$ of $\sim-0.6 \mathrm{dex}$ (see Fig. 9). It is known that there is a large spread in the solar neighbourhood metallicity distribution (see e.g. Haywood 2001) with $-0.3<$ $[\mathrm{Fe} / \mathrm{H}]_{\mathrm{RSFD}}^{\text {index }}<0$; an offset of $\sim 0.3$ dex with respect to the solar neighbourhood sample of LW is apparent. The relation of RSFD is based on the calibration of globular clusters and thus is biased towards metal-poor stars. At higher metallicities the $\mathrm{CO}$ band reaches a plateau and becomes insensitive to changes in $[\mathrm{Fe} / \mathrm{H}]_{\mathrm{RSFD}}^{\mathrm{index}}$. Since solution 1 has a strong dependence on $E W(\mathrm{CO})$, it will therefore underestimate $[\mathrm{Fe} / \mathrm{H}]_{\mathrm{RSFD}}^{\text {index }}$ for larger metallicities. However, Fig. 9 shows that our ISOGAL sample as well as the sample of RSFD is $\sim 0.4$ dex metal richer than the solar neighbourhood sample of LW. Haywood (2001) gives a modelled and an observed age-metallicity relation (see their Fig. 14a). According to that, a $[\mathrm{Fe} / \mathrm{H}]$ of -0.6 dex corresponds to ages of about $8-9$ Gyr and thus for AGB stars an initial mass of the order of one solar mass. So, according to the age-metallicity relation, the solar neighbourhood sample of LW have initial masses around $1 M_{\odot}$, in agreement with mass estimates derived from pulsation periods (see Table 8 of LW). The difference in the metallicity distribution in the solar neighbourhood and in the Bulge results probably from both different mean stellar ages and different mean age-metallicity relations due to different star formation histories and chemical evolution histories. However, the validity of such age-metallicity relations is still on debate (see e.g. the recent review of Freeman \& Bland-Hawthorn 2003).

The accurate determination of mean stellar metallicities is essential for constraining models of star formation and chemical evolution in the Bulge. Our mean $[\mathrm{Fe} / \mathrm{H}]$ value is consistent with previous chemical abundance studies of the galactic Bulge (see e.g. McWilliam \& Rich 1994; Minniti et al. 1995; Houdashelt 1996; Frogel et al. 1999, RSFD) where the metallicity peaks $\sim-0.3$ dex. Moreover, Molla et al. (2000) model 
the evolution of the Galactic Bulge which assumes a dissipative collapse of the gas from a protogalaxy or halo to form the Bulge and the disk. They predict a mean stellar Bulge metallicity $[\mathrm{Fe} / \mathrm{H}] \sim-0.2$ dex with a dispersion of \pm 0.40 dex.

A chemical abundance gradient in the Bulge is one characteristics of a Bulge formed by a dissipative collapse. The determination of the metallicity gradient in the central regions of our Galaxy is important for testing models of Galaxy formation. Up to now, only a few studies of the metallicity gradient exist in the inner Bulge $\left(|b|<3^{0}\right)$. Frogel et al. (1999) found a metallicity gradient based on near-IR photometric data while RSFD could not find any evidence. The dissipative collapse model of Molla et al. (2000) predict a steep metallicity gradient $\left(-0.8 \mathrm{dex} \mathrm{kpc}^{-1}\right)$ in the inner Bulge $(R<500 \mathrm{pc})$ which is not supported by the data of RSFD. Our sample of near-IR spectra presented here, which agrees with the metallicity distribution of RSFD, seems to confirm the absence of a metallicity gradient in the Bulge.

We want to stress that our data set is not sufficient to do a large statistical analysis of chemical abundances in the galactic Bulge. Therefore a more detailed discussion of the implications of the results is beyond the scope of this paper; a large observational program is necessary.

It is obvious from the discussion above that only realistic models of $\mathrm{M}$ giants including the effects of metallicity can enable us to make a quantitative determination of their metallicity. First tests of synthetic spectra based on hydrostatic MARCS model atmospheres (in collaboration with B. Aringer) and including a complete atomic line list already show some promising results (Aringer et al. 2002). However, for AGB stars (the majority of our stars) hydrodynamical models would be more appropriate (see e.g. Höfner 1999; Aringer et al. 2000).

\section{Conclusions}

We have studied a sample of 107 near-IR spectra of ISOGAL sources with excesses at 7 and $15 \mu \mathrm{m}$ in the innermost parts of the galactic Bulge where the interstellar extinction is high and clumpy. We have shown that using the molecular bands of $\mathrm{CO}$ and $\mathrm{H}_{2} \mathrm{O}$, together with the bolometric magnitudes and the interstellar extinction values, one can reasonably well separate AGB stars, red giants, supergiants and young stellar objects. We have found four supergiant candidates, twelve red giant candidates and nine YSOs, while the rest are probably variable AGB stars. We have used the most recent $K_{0}-[15]$ vs. $\dot{M}$ relation by Jeong et al. (2002) which is based on a self-consistent time dependent model of dust formation in AGB stars to determine mass-loss rates. We emphasize that the color $\dot{M}$ relation has been determined from a model of AGB/LPVs and gives only an indication of the mass-loss rate. From our sample, OH/IR stars cover mass-loss rates ranging from $-6<\log \dot{M}<-4.5$ while LPVs range between $-6.5<$ $\log \dot{M}<-5$. Our red giant candidates show mass-loss rates in the range between $-8<\log \dot{M}<-6$. However, this selection is biased in favor of large $\dot{M}$. A comparison with mass-loss rates of red giants in globular clusters (Origlia \& Ferrero 2002) shows that our mass-loss range is much broader towards larger values of $\dot{M}$. However, the uncertainty in the determination of $\dot{M}$ is rather large.

While the quantity $\log [E W(\mathrm{CO}) /(E W(\mathrm{Na})+E W(\mathrm{Ca}))]$ of $\mathrm{RDF}$ is a good discriminator between giants and dwarfs, it cannot be used to separate supergiants and LPVs from red giants. However, the dispersion is much narrower for $\mathrm{M}$ giants than for variable AGB stars.

We have used the metallicity index $[\mathrm{Fe} / \mathrm{H}]_{\mathrm{RSFD}}^{\text {index }}$ of RSFD, determined from the line strengths of $\mathrm{CO}, \mathrm{NaI}$ and $\mathrm{CaI}$, to estimate the metallicity of the stellar population. Our mean $[\mathrm{Fe} / \mathrm{H}]_{\mathrm{RSFD}}^{\mathrm{index}}$ is -0.25 dex with a dispersion of \pm 0.40 dex which is in agreement with the values obtained by RSFD and supports the argument of RSFD that there is no metallicity gradient in the Bulge. Our mean $[\mathrm{Fe} / \mathrm{H}]_{\mathrm{RSFD}}^{\mathrm{index}}$ is consistent with previous abundance studies of the galactic Bulge and with the multiphase evolution models of Molla et al. (2000) which assume a dissipative collapse of a protogalaxy to form the Bulge and the galactic disk. Our results confirm that even if the metallicity index of RSFD is only valid for M giants, it can be used to estimate an average metallicity valid over a whole pulsation cycle. This is an important result for future stellar population studies using multi-fiber spectrographs (such as GIRAFFE, KMOS, etc.). However, there is a pressing need for a grid of realistic models of $\mathrm{M}$ giants with different $[\mathrm{Fe} / \mathrm{H}]$ to facilitate a quantitative determination of metallicities, as well as further modeling of LPV/AGB stars.

Acknowledgements. We want to thank I. S. Glass, J. Blommaert, J. van Loon and M. Messineo for their fruitful comments and discussions. MS is supported by the Fonds zur Förderung der wissenschaftlichen Forschung (FWF), Austria, under the project number J1971-PHY.

This research is supported by the project 1910-1 of Indo-French Center for the Promotion of Advanced Research (CEFIPRA).

The DENIS project is supported, in France by the Institut National des Sciences de l'Univers, the Education Ministry and the Centre National de la Recherche Scientifique, in Germany by the State of Baden-Würtemberg, in Spain by the DGICYT, in Italy by the Consiglio Nazionale delle Ricerche, in Austria by the Fonds zur Förderung der wissenschaftlichen Forschung und Bundesministerium für Wissenschaft und Forschung.

This research has made use of the Simbad database, operated at CDS, Strasbourg, France.

\section{Appendix A: Tables}

\section{Appendix B: Near-IR spectra}

The spectra were dereddened using the extinction curve by Mathis et al. (1990) and the DENIS extinction map (Schultheis et al. 1999), see Sect. 3.1. The flux densities per unit wavelength are normalized at $2.28 \mu \mathrm{m}$. The spectra are electronically available at CDS. Strong telluric bands are seen between 1.8 and $1.9 \mu \mathrm{m}$. 
Table A.1. Coordinates (J2000), magnitudes (DENIS, ISOGAL), $A_{V}$ values, equivalent widths (in $\AA$ ) of ${ }^{12} \mathrm{CO}(2,0), \mathrm{NaI}, \mathrm{CaI}, \mathrm{H}_{2} \mathrm{O}, M_{\text {bol }}, \log \dot{M}$.

\begin{tabular}{|c|c|c|c|c|c|c|c|c|c|c|c|c|c|c|}
\hline Name & Right ascension & Declination & $\bar{J}$ & $\overline{K_{\mathrm{S}}}$ & [7] & [15] & $\overline{A_{V}}$ & $12^{\mathrm{CO}}(2,0)$ & $\mathrm{NaI}$ & $\overline{\mathrm{CaI}}$ & $\overline{\mathrm{H}_{2} \mathrm{O}}$ & $\overline{M_{\text {bol }}}$ & $\log \dot{M}$ & Type \\
\hline A3 & 174423.8 & -29855.3 & 14.57 & 10.03 & 8.74 & 6.43 & 19.60 & 19.48 & 4.93 & 1.87 & -35.78 & -3.25 & -6.69 & RGB \\
\hline A4 & 174430.4 & -29715.5 & 15.14 & 9.23 & 4.77 & 3.93 & 19.10 & 9.83 & 2.44 & -0.50 & -261.11 & -4.32 & -5.46 & AGB \\
\hline A5 & 174431.7 & -29620.6 & 13.80 & 8.50 & 5.08 & 2.92 & 19.70 & 21.04 & 2.26 & -0.74 & -666.39 & -4.83 & -5.36 & AGB \\
\hline A6 & 174431.8 & -291710.5 & - & 9.58 & 5.58 & 4.04 & 24.50 & 16.88 & 3.94 & 0.23 & -157.16 & -4.52 & -5.50 & AGB \\
\hline A7 & 174434.4 & -291038.5 & 13.41 & 8.04 & 4.05 & 1.76 & 21.60 & 21.93 & 4.10 & -0.11 & -386.72 & -5.71 & -5.17 & $\mathrm{OH} / \mathrm{IR}$ \\
\hline A8 & 174435.7 & -29135.4 & 15.20 & 8.68 & 4.83 & 3.15 & 25.10 & 13.75 & 2.17 & -0.51 & -259.69 & -5.04 & -5.52 & AGB \\
\hline A9 & 174436.4 & -29925.2 & - & 9.67 & 4.20 & 2.62 & 22.30 & 20.74 & 4.88 & 2.41 & -23.84 & -5.08 & -4.99 & AGB \\
\hline A10 & 174439.7 & -291645.9 & - & 10.99 & 4.50 & 2.35 & 25.00 & 19.09 & 5.25 & 3.61 & -13.92 & -4.45 & -4.75 & $\mathrm{OH} / \mathrm{IR}$ \\
\hline $\mathrm{A} 12$ & 174444.5 & -29538.3 & - & 11.18 & 3.57 & 2.05 & 20.50 & 12.99 & 1.29 & -2.11 & -181.29 & -6.03 & -4.65 & $\mathrm{OH} / \mathrm{IR}$ \\
\hline $\mathrm{A} 13$ & 174444.6 & -29733.6 & 15.84 & 9.26 & 5.90 & 4.35 & 19.40 & 8.38 & 2.30 & -1.37 & -434.36 & -3.74 & -5.64 & AGB \\
\hline A14 & 174448.0 & -29649.8 & 15.24 & 9.34 & 5.03 & 2.85 & 20.50 & 20.94 & 4.57 & 1.94 & -120.23 & -4.36 & -5.10 & $\mathrm{OH} / \mathrm{IR}$ \\
\hline A16 & 174448.6 & -29013.2 & 15.62 & 9.89 & 8.16 & 99.99 & 21.50 & 21.04 & 6.26 & 1.35 & -54.97 & -3.14 & - & RGB \\
\hline A17 & 174449.1 & -291954.2 & 11.08 & 6.17 & 3.41 & 1.31 & 25.40 & 20.58 & 0.78 & -0.24 & -52.70 & -8.06 & -5.89 & supergiant \\
\hline A18 & 174449.5 & -29315.9 & 15.08 & 9.42 & 6.92 & 5.21 & 21.60 & 29.17 & 6.16 & 1.31 & -336.94 & -3.76 & -6.20 & LPV \\
\hline A19 & 174450.3 & -291921.5 & 14.58 & 9.01 & 6.92 & 5.34 & 24.80 & 19.80 & 5.21 & 1.41 & -184.69 & -4.65 & -7.06 & AGB \\
\hline $\mathrm{A} 20$ & 174452.7 & -291411.1 & - & 9.61 & 7.62 & 5.13 & 24.50 & 20.76 & 5.47 & 3.40 & -27.37 & -4.01 & -6.13 & AGB \\
\hline $\mathrm{A} 21$ & 174452.9 & -2976.7 & - & 9.30 & 5.38 & 3.73 & 28.50 & 18.84 & 4.21 & 1.63 & -144.06 & -4.98 & -5.60 & AGB \\
\hline A22 & 174453.1 & -285946.5 & - & 10.78 & 8.76 & 6.82 & 19.80 & 18.94 & 5.42 & 2.91 & -19.26 & -2.48 & -6.32 & RGB \\
\hline $\mathrm{A} 23$ & 174454.2 & -291344.9 & 14.84 & 8.58 & 4.89 & 3.23 & 24.50 & 22.34 & 3.70 & - & -543.70 & -5.08 & -5.59 & $\mathrm{OH} / \mathrm{IR}$ \\
\hline A24 & 174454.7 & -29359.0 & - & 10.70 & 5.13 & 3.29 & 22.10 & 7.52 & 0.19 & -0.95 & -277.07 & -3.80 & -4.92 & AGB \\
\hline A25 & 174455.5 & -29143.4 & 14.34 & 8.53 & 5.48 & 3.91 & 19.80 & 20.48 & 3.54 & -0.76 & -623.25 & -4.52 & -5.82 & AGB \\
\hline A26 & 174456.8 & -291020.3 & 15.77 & 9.05 & 6.11 & 4.41 & 25.30 & 19.99 & 3.76 & -1.08 & -526.70 & -4.36 & -6.04 & LPV \\
\hline A27 & 174457.0 & -29557.3 & 15.25 & 10.11 & 4.48 & 1.65 & 25.30 & 19.20 & 4.29 & 3.11 & -8.01 & -5.07 & -4.78 & AGB \\
\hline A28 & 174457.1 & -291524.7 & - & 8.84 & 5.30 & 3.51 & 20.30 & 17.13 & 1.49 & -1.44 & -473.84 & -4.67 & -5.47 & AGB \\
\hline A29 & 174457.8 & -292042.5 & - & 10.65 & 4.66 & 2.45 & 23.40 & 8.98 & -0.05 & -1.75 & 129.52 & -4.32 & -4.80 & $\mathrm{OH} / \mathrm{IR}$ \\
\hline A30 & 174457.9 & -2946.5 & 14.64 & 9.15 & 7.14 & 5.46 & 22.30 & 23.18 & 5.60 & 2.32 & -112.56 & -4.13 & -6.80 & AGB \\
\hline A31 & 174458.9 & -29910.8 & - & 8.19 & 4.73 & 2.99 & 24.40 & 17.21 & 1.34 & -0.77 & -332.12 & -5.63 & -5.66 & LPV \\
\hline A33 & 174459.5 & -29164.6 & - & 10.78 & 4.08 & 0.50 & 20.30 & -2.28 & 0.42 & -1.24 & 1.78 & 2504.72 & - & YSO \\
\hline A34 & 174459.6 & -291115.4 & 13.43 & 7.95 & 5.50 & 3.75 & 23.80 & 22.46 & 3.96 & -1.06 & -505.14 & -5.64 & -6.33 & LPV \\
\hline A35 & 17451.0 & -29114.9 & - & 9.43 & 5.90 & 4.27 & 19.80 & 12.08 & 1.27 & - & -430.36 & -4.18 & -5.53 & LPV \\
\hline A36 & 17451.7 & -29250.0 & 13.83 & 8.23 & 4.91 & 3.26 & 18.50 & 20.32 & 2.64 & -0.16 & -694.69 & -4.83 & -5.58 & $\mathrm{OH} / \mathrm{IR}$ \\
\hline A38 & 17454.8 & -29124.9 & 13.94 & 8.52 & 5.52 & 3.86 & 23.10 & 7.78 & 0.65 & 0.69 & -498.84 & -5.06 & -5.93 & $\mathrm{OH} / \mathrm{IR}$ \\
\hline A39 & 17454.8 & -29548.5 & 13.69 & 7.85 & 4.86 & 3.31 & 23.10 & 22.72 & 3.34 & 0.20 & -347.86 & -5.56 & -6.01 & LPV \\
\hline $\mathrm{A} 40$ & 17454.9 & -291146.8 & 15.24 & 9.01 & 4.27 & 1.97 & 23.50 & -1.61 & 0.58 & -0.18 & 77.81 & -5.14 & - & YSO \\
\hline A41 & 17455.3 & -29135.8 & 13.89 & 8.46 & 5.47 & 3.75 & 20.60 & 17.11 & 2.32 & 0.85 & -536.87 & -4.77 & -5.79 & LPV \\
\hline A43 & 17457.0 & -29334.2 & 13.94 & 8.56 & 4.86 & 2.73 & 24.80 & 18.78 & -0.14 & 0.36 & -993.36 & -5.46 & -5.39 & $\mathrm{OH} / \mathrm{IR}$ \\
\hline A45 & 17459.8 & -29517.8 & - & 10.87 & 7.82 & 6.26 & 22.80 & 17.07 & 3.82 & 1.12 & -86.09 & -2.82 & -5.95 & RGB \\
\hline A47 & 174510.8 & -29712.2 & 15.59 & 9.61 & 7.69 & 4.82 & 22.80 & 18.75 & 4.46 & 2.02 & -52.85 & -3.61 & -5.83 & AGB \\
\hline A49 & 174512.2 & -29425.2 & - & 8.72 & 4.85 & 3.28 & 22.30 & 21.14 & 3.20 & -0.38 & -643.49 & -5.19 & -5.48 & LPV \\
\hline A50 & 174513.5 & -29526.7 & 13.17 & 7.62 & 4.56 & 2.98 & 23.90 & 16.59 & 1.52 & -0.06 & -629.52 & -6.03 & -5.98 & LPV \\
\hline A51 & 174514.0 & -29 1527.4 & 15.93 & 10.31 & 3.47 & 1.59 & 24.00 & 15.75 & 3.32 & 2.54 & -54.14 & 2504.72 & -4.73 & $\mathrm{OH} / \mathrm{IR}$ \\
\hline A52 & 174514.3 & -29720.8 & - & 10.60 & 4.14 & 2.40 & 22.90 & 8.78 & 1.01 & 0.12 & -318.86 & -4.94 & -4.79 & $\mathrm{OH} / \mathrm{IR}$ \\
\hline
\end{tabular}




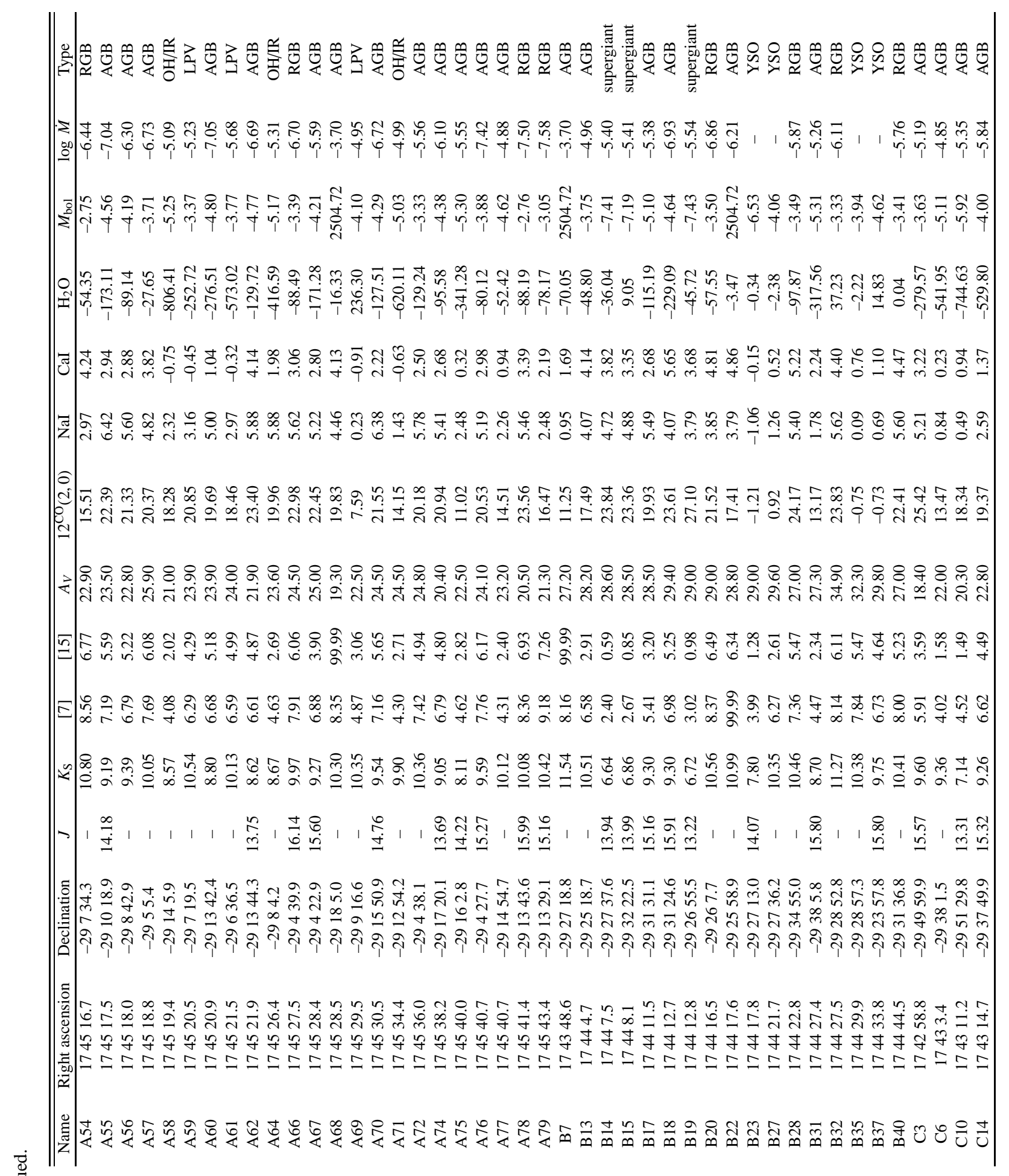


Table A.1. continued.

\begin{tabular}{|c|c|c|c|c|c|c|c|c|c|c|c|c|c|c|}
\hline Name & Right ascension & Declination & $\bar{J}$ & $\overline{K_{\mathrm{S}}}$ & [7] & [15] & $\overline{\overline{A_{V}}}$ & $12^{\mathrm{CO}}(2,0)$ & $\mathrm{NaI}$ & $\mathrm{CaI}$ & $\overline{\mathrm{H}_{2} \mathrm{O}}$ & $M_{\text {bol }}$ & $\overline{l o g} \dot{M}$ & Type \\
\hline C19 & 174319.3 & -295028.9 & 13.92 & 10.98 & 6.53 & 3.80 & 22.50 & -3.64 & -0.58 & 0.12 & -50.59 & -4.24 & - & YSO \\
\hline $\mathrm{C} 23$ & 174324.6 & -295312.4 & 14.33 & 8.56 & 6.89 & 4.82 & 23.60 & 25.90 & 5.16 & 4.89 & -237.00 & -4.79 & -6.84 & AGB \\
\hline $\mathrm{C} 25$ & 174325.0 & -294123.0 & - & 9.32 & 5.37 & 3.19 & 25.00 & 16.35 & 3.10 & 3.17 & -223.55 & -4.57 & -5.29 & AGB \\
\hline $\mathrm{C} 45$ & 174344.2 & -293826.1 & 15.61 & 9.75 & 7.50 & 4.33 & 23.80 & 25.52 & 5.19 & 3.28 & -279.99 & -3.72 & -5.53 & AGB \\
\hline D5 & 174227.3 & -295417.8 & 13.28 & 8.04 & 6.50 & 4.44 & 20.30 & 13.49 & 3.05 & 2.79 & -364.41 & -5.02 & -6.75 & AGB \\
\hline D6 & 174228.0 & -295614.6 & 13.90 & 10.55 & 3.87 & 0.13 & 24.30 & -1.00 & 0.03 & 1.44 & -57.42 & 2504.72 & - & YSO \\
\hline D7 & 174229.9 & -30115.9 & - & 10.97 & 3.36 & -0.06 & 22.40 & 0.87 & 1.20 & 1.76 & -82.31 & 2504.72 & - & YSO \\
\hline D11 & 174244.4 & -295836.1 & 14.03 & 7.94 & 4.60 & 2.39 & 26.00 & 12.16 & 1.85 & 0.66 & -544.74 & -5.89 & -5.54 & AGB \\
\hline D13 & 174247.7 & -295625.5 & - & 9.56 & 6.74 & 4.40 & 27.90 & 22.36 & 5.19 & 4.34 & -115.25 & -4.40 & -5.80 & AGB \\
\hline $\mathrm{D} 22$ & 174329.8 & -30127.3 & 12.17 & 7.89 & 5.73 & 3.59 & 19.00 & 21.64 & 1.69 & 2.07 & -1020.49 & -5.49 & -5.99 & AGB \\
\hline E4 & 174313.0 & -29211.4 & - & 7.22 & 5.32 & 4.33 & 23.30 & 20.36 & 4.41 & 2.70 & -221.63 & -6.38 & -8.66 & AGB \\
\hline E5 & 174315.5 & -292458.7 & 11.51 & 6.58 & 4.87 & 4.07 & 25.70 & 23.21 & 5.36 & 3.04 & -109.75 & -7.64 & -11.67 & AGB \\
\hline E13 & 174328.2 & -291741.7 & 11.33 & 6.93 & 5.21 & 4.67 & 22.70 & 20.18 & 4.05 & 2.13 & -193.37 & -7.05 & -12.24 & AGB \\
\hline E20 & 174339.2 & -292244.5 & - & 10.61 & 6.61 & 4.24 & 25.50 & 19.69 & 3.39 & 1.65 & -162.79 & -3.31 & -5.22 & AGB \\
\hline E51 & 174435.0 & -29435.5 & - & 9.09 & 1.30 & -0.39 & 20.10 & 10.11 & 1.60 & 0.61 & -297.85 & -8.22 & -4.61 & $\mathrm{OH} / \mathrm{IR}$ \\
\hline F13 & 174228.0 & -293746.4 & 15.21 & 9.87 & 5.23 & 3.01 & 17.00 & 20.61 & 2.00 & 1.12 & -763.02 & -3.87 & -4.96 & AGB \\
\hline F14 & 174239.5 & -294327.3 & 15.36 & 9.91 & 4.95 & 3.13 & 21.50 & 18.15 & 2.57 & 3.08 & -186.75 & -4.24 & -5.04 & AGB \\
\hline $\mathrm{F} 23$ & 174036.3 & -294913.7 & 11.51 & 8.18 & 5.71 & 2.21 & 9.80 & 10.91 & 0.91 & 2.72 & -597.37 & -4.49 & -5.06 & AGB \\
\hline G35 & 174316.4 & -301310.7 & 14.14 & 10.98 & 4.95 & 2.91 & 16.30 & 19.06 & 1.77 & 0.82 & -340.69 & -3.94 & -4.75 & AGB \\
\hline G46 & 17447.5 & -30741.3 & 14.09 & 8.63 & 5.32 & 3.46 & 20.60 & 18.51 & 3.51 & 1.45 & -506.89 & -4.69 & -5.55 & AGB \\
\hline H01 & 173053.2 & -334021.7 & 15.72 & 10.53 & 5.53 & 2.92 & 10.80 & 13.11 & 2.50 & 1.43 & -492.72 & -3.36 & -4.77 & AGB \\
\hline
\end{tabular}



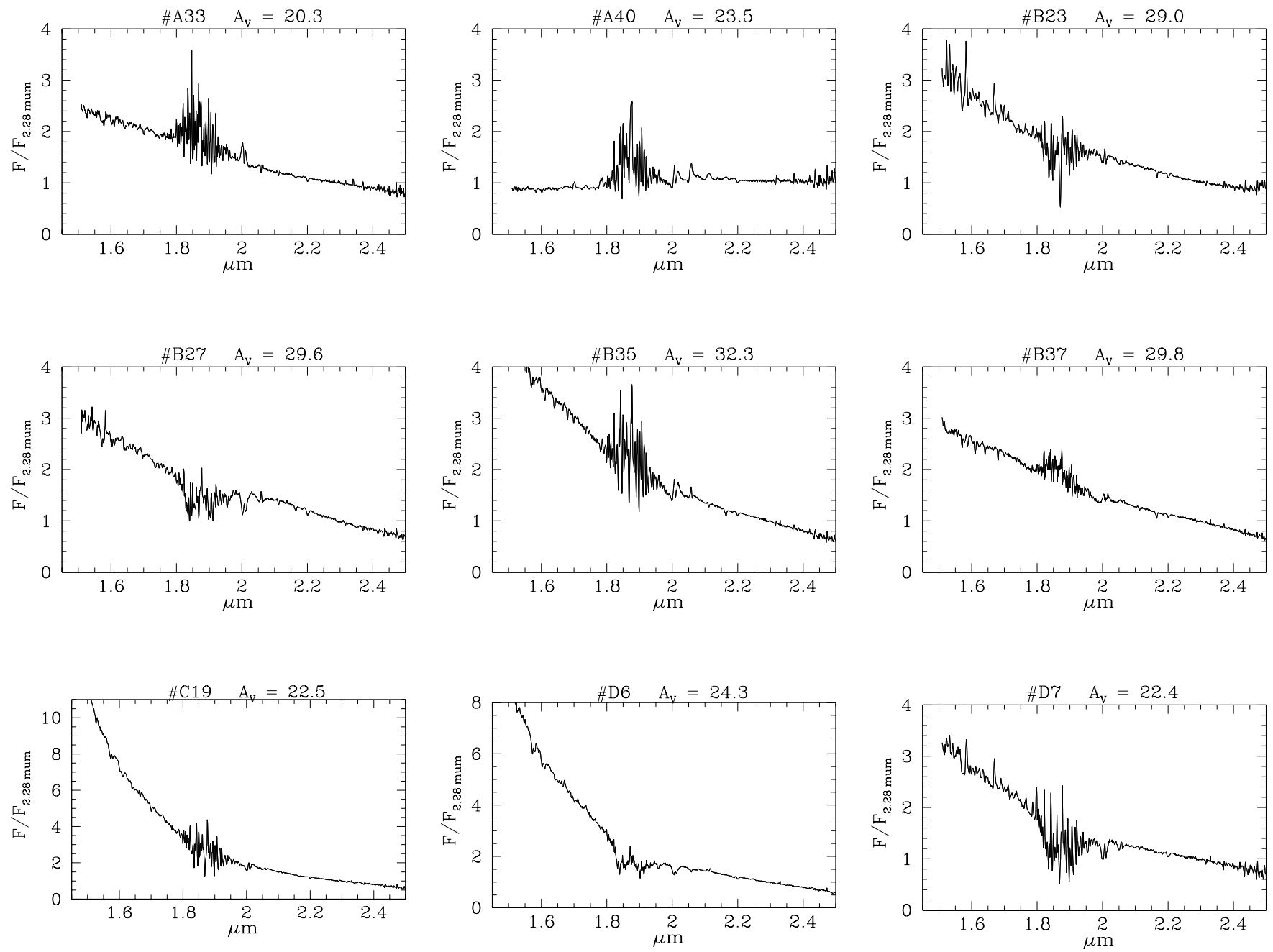

Fig. B.1. Young stellar objects.
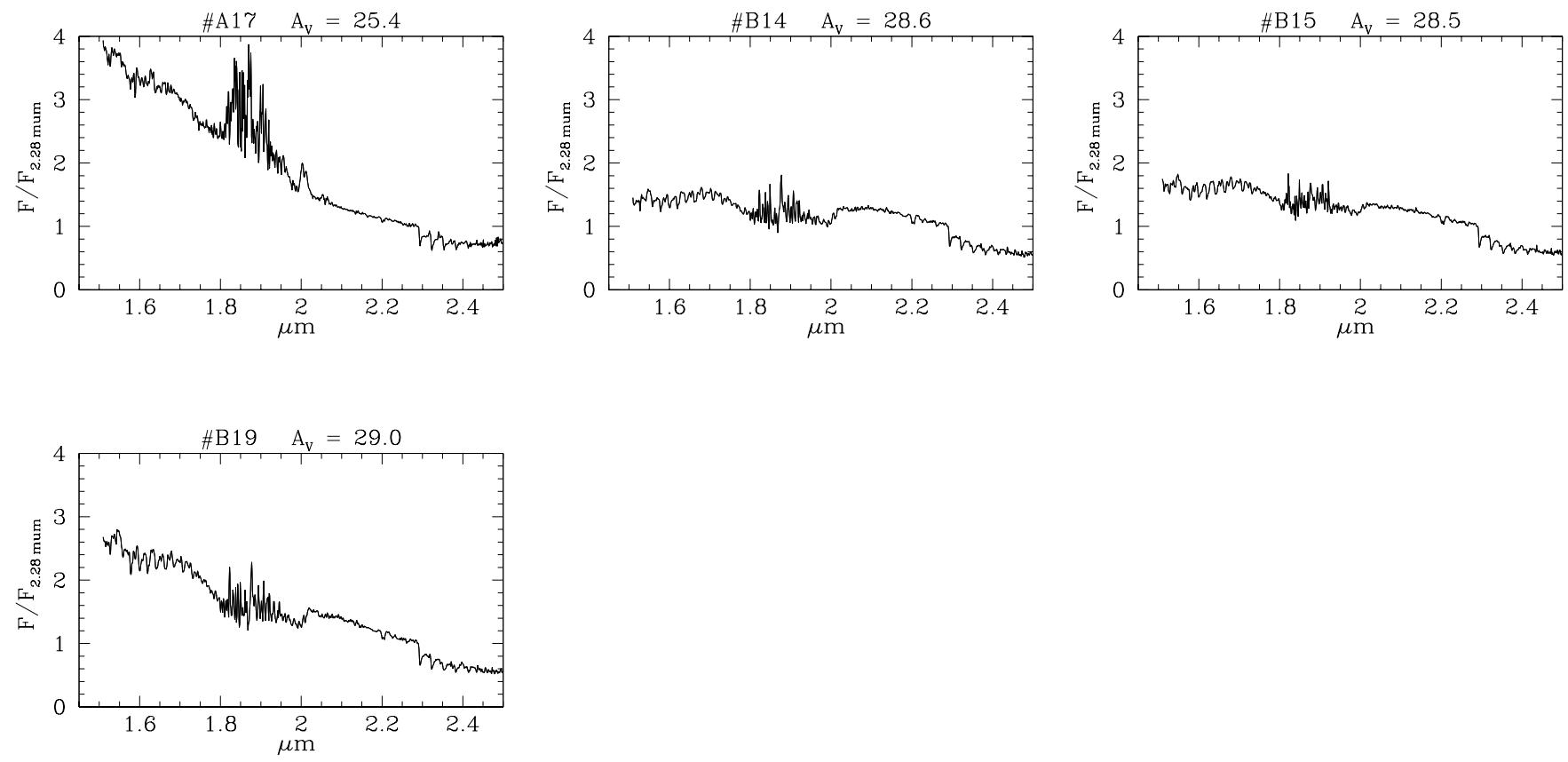

Fig. B.2. Supergiants candidates. 

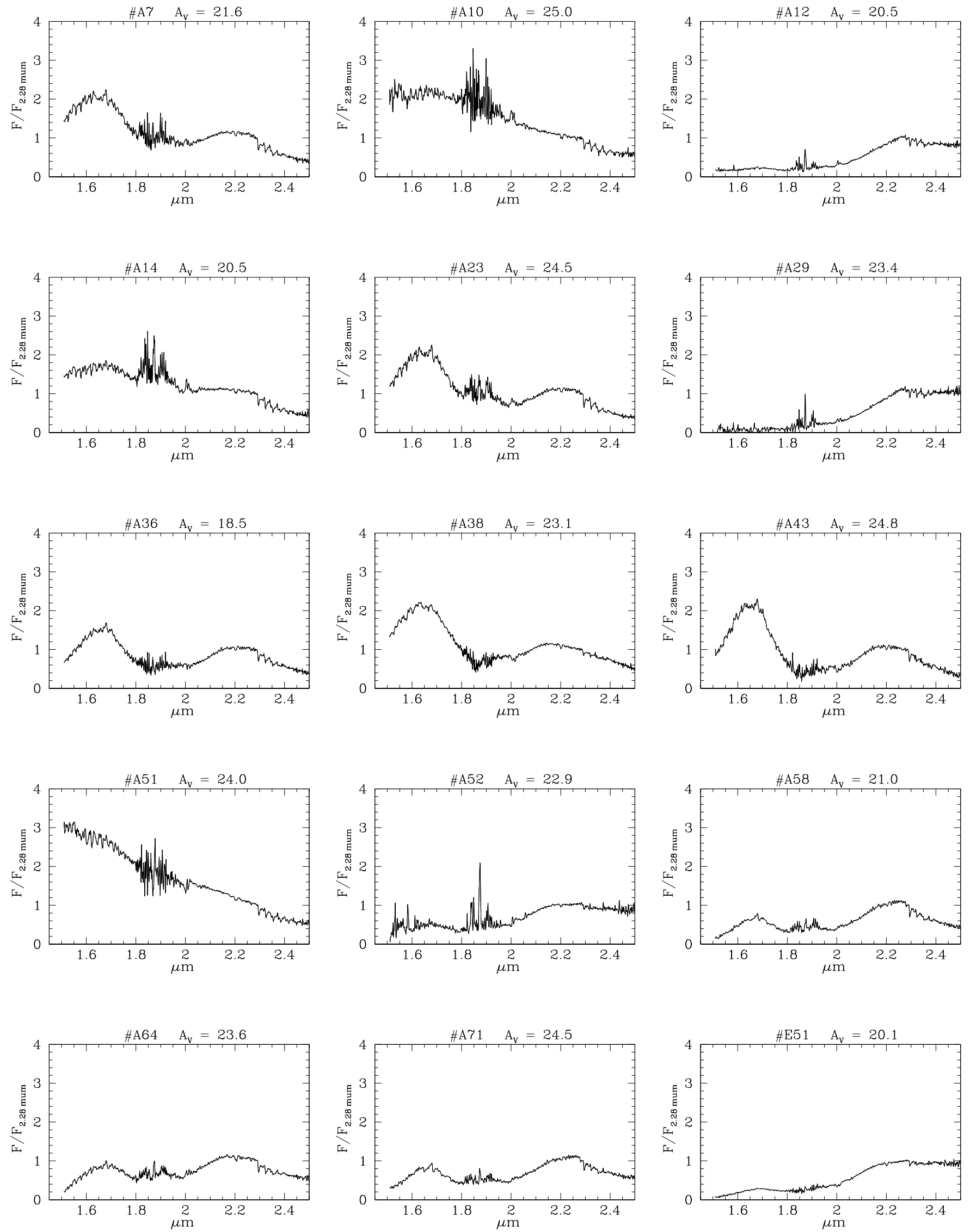

Fig. B.3. OH/IR stars (Wood et al. 1998). 

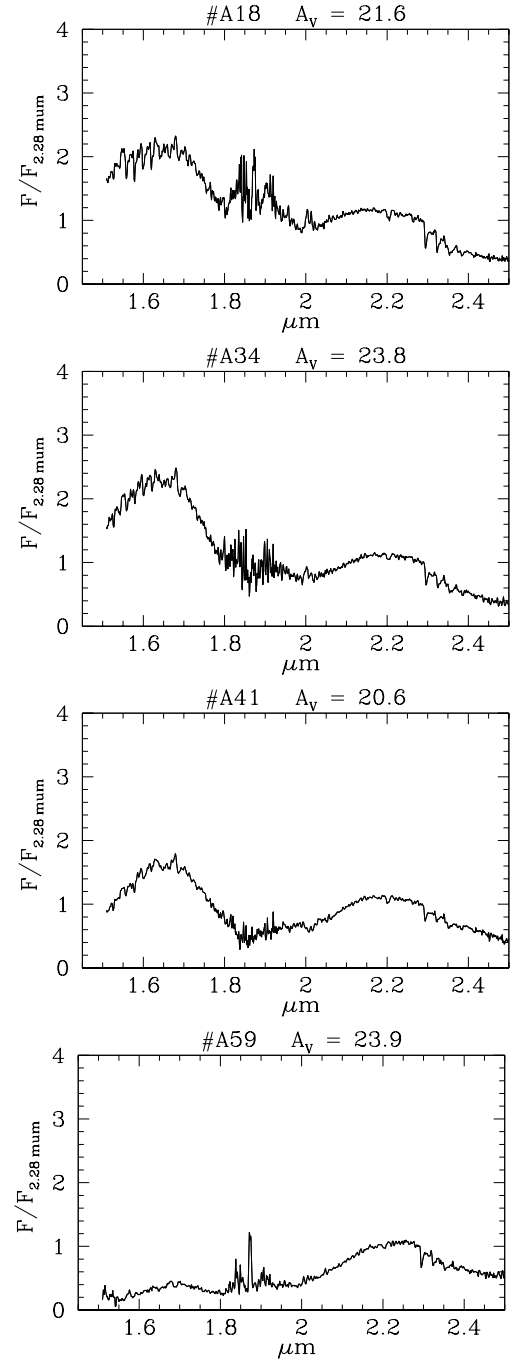

Fig. B.4. Long period Variables (Glass et al. 2001).
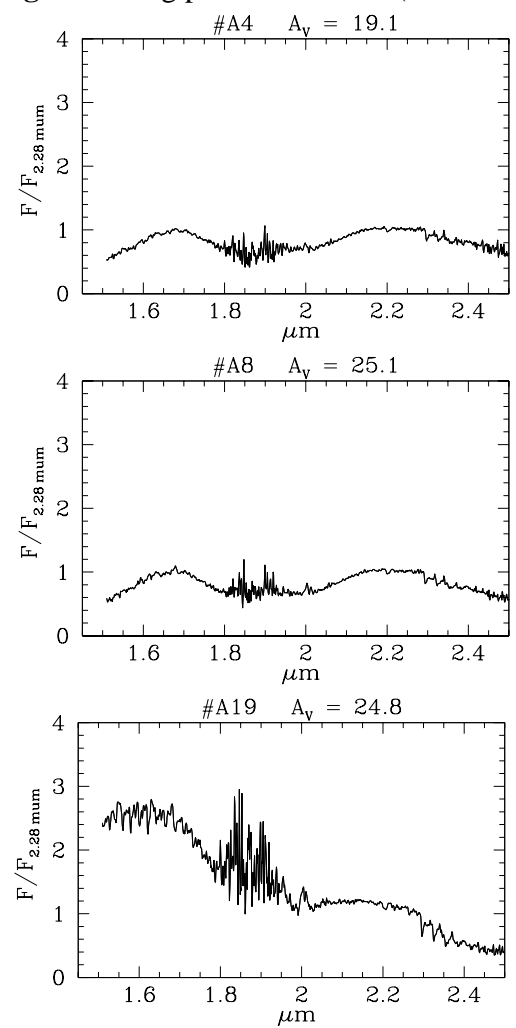

Fig. B.5. AGB star candidates.
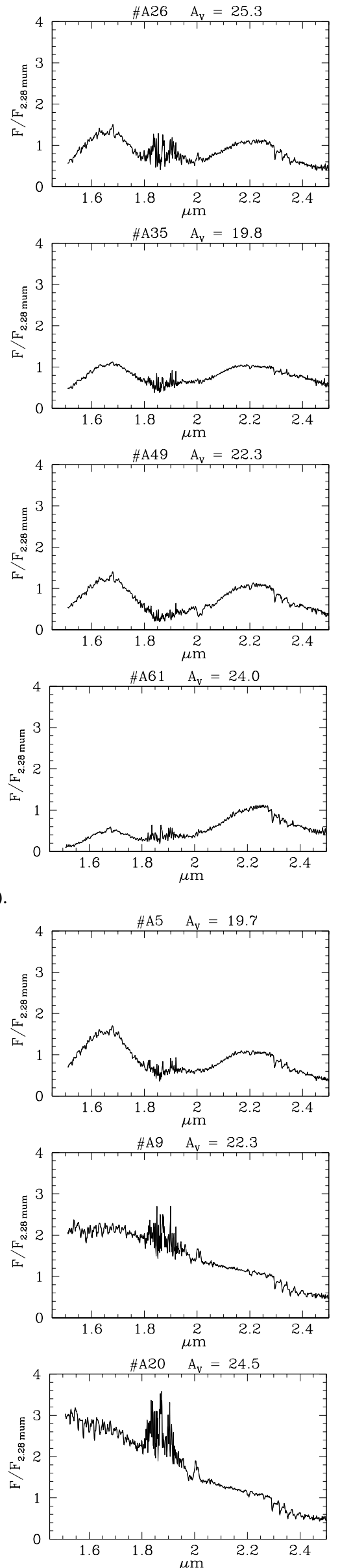
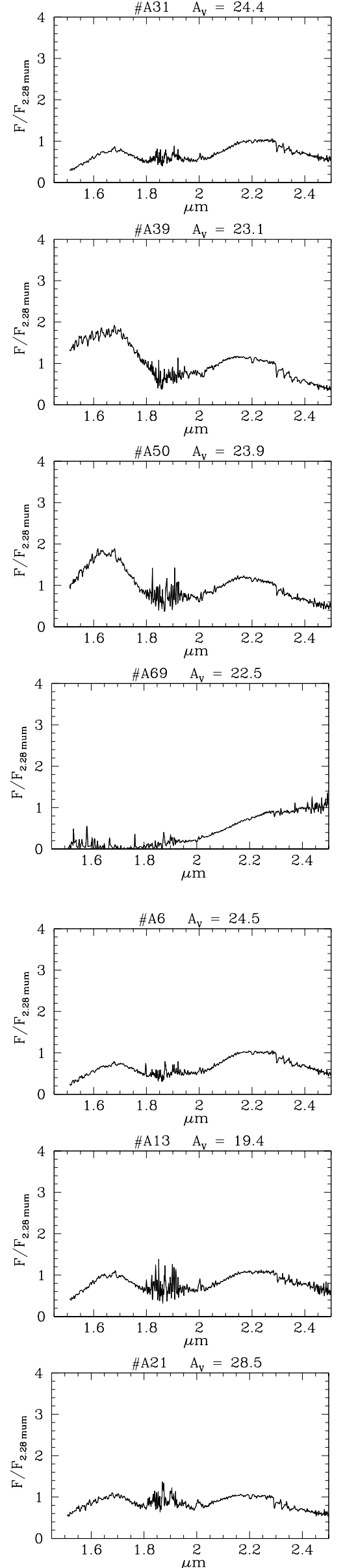

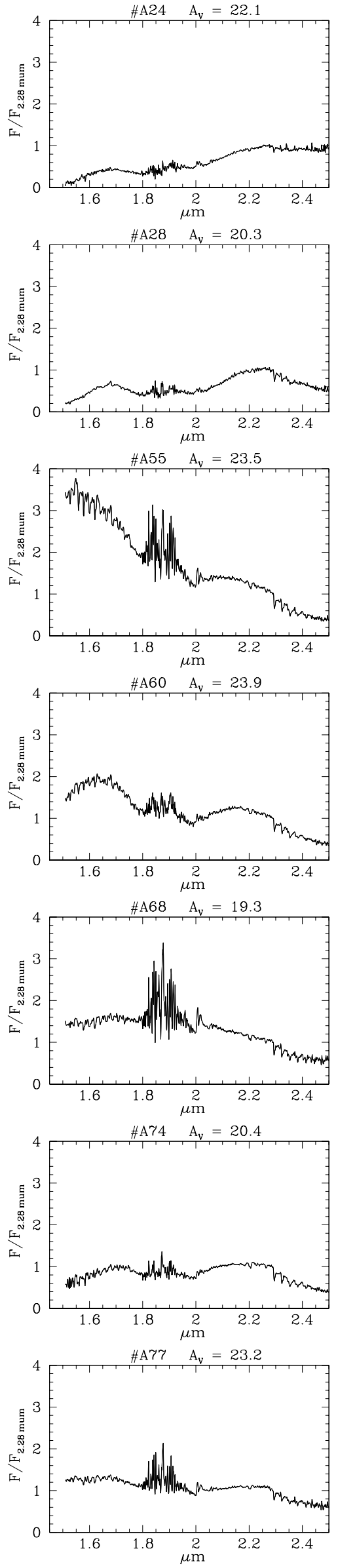

Fig. B.5. continued.
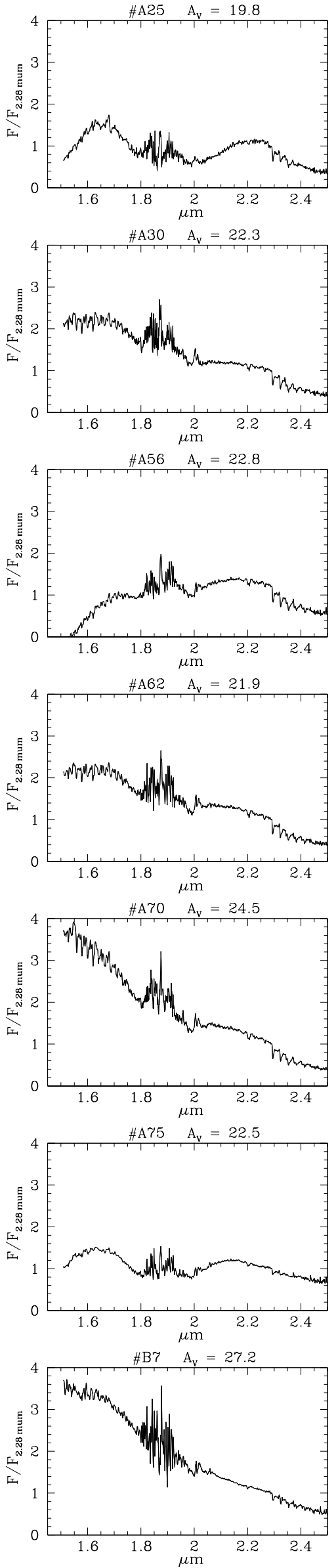
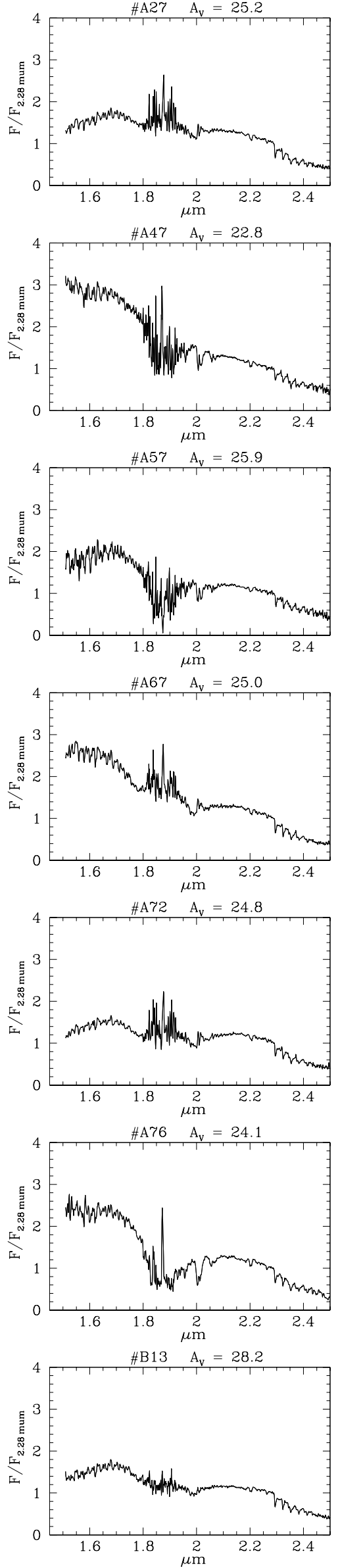

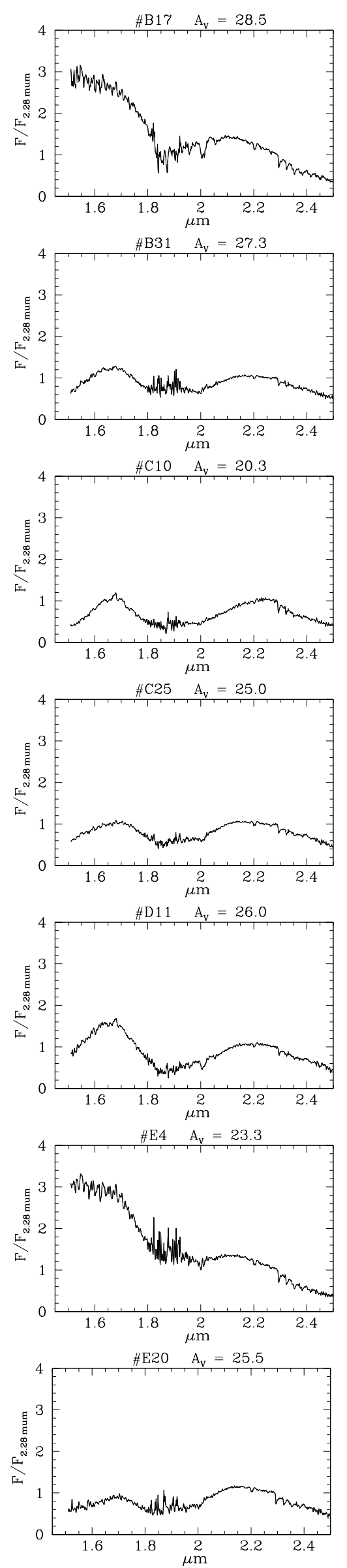

Fig. B.5. continued.
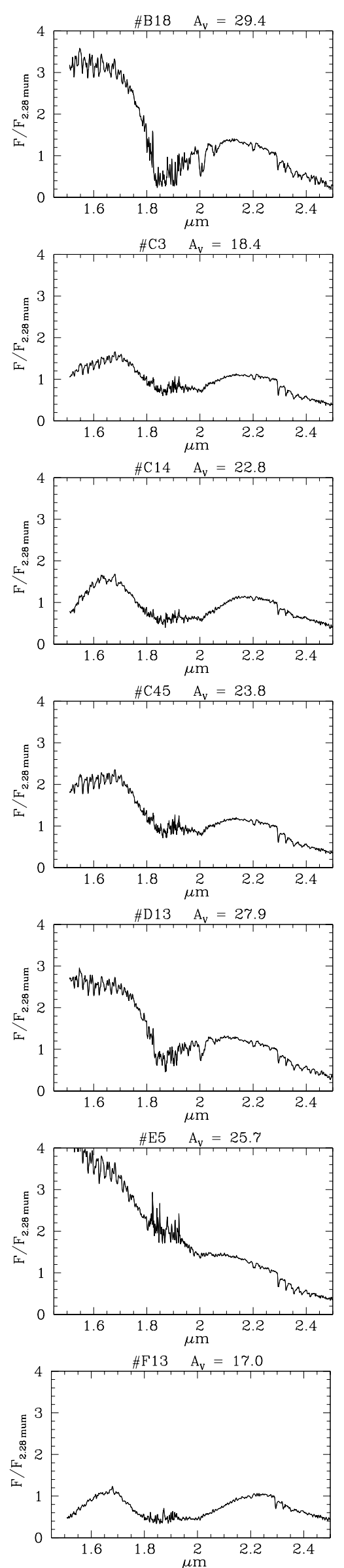
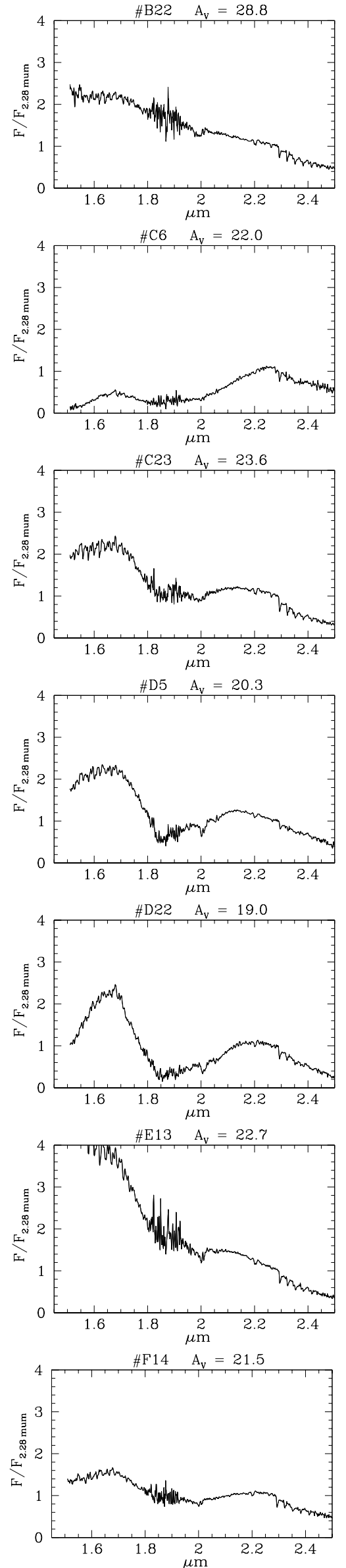

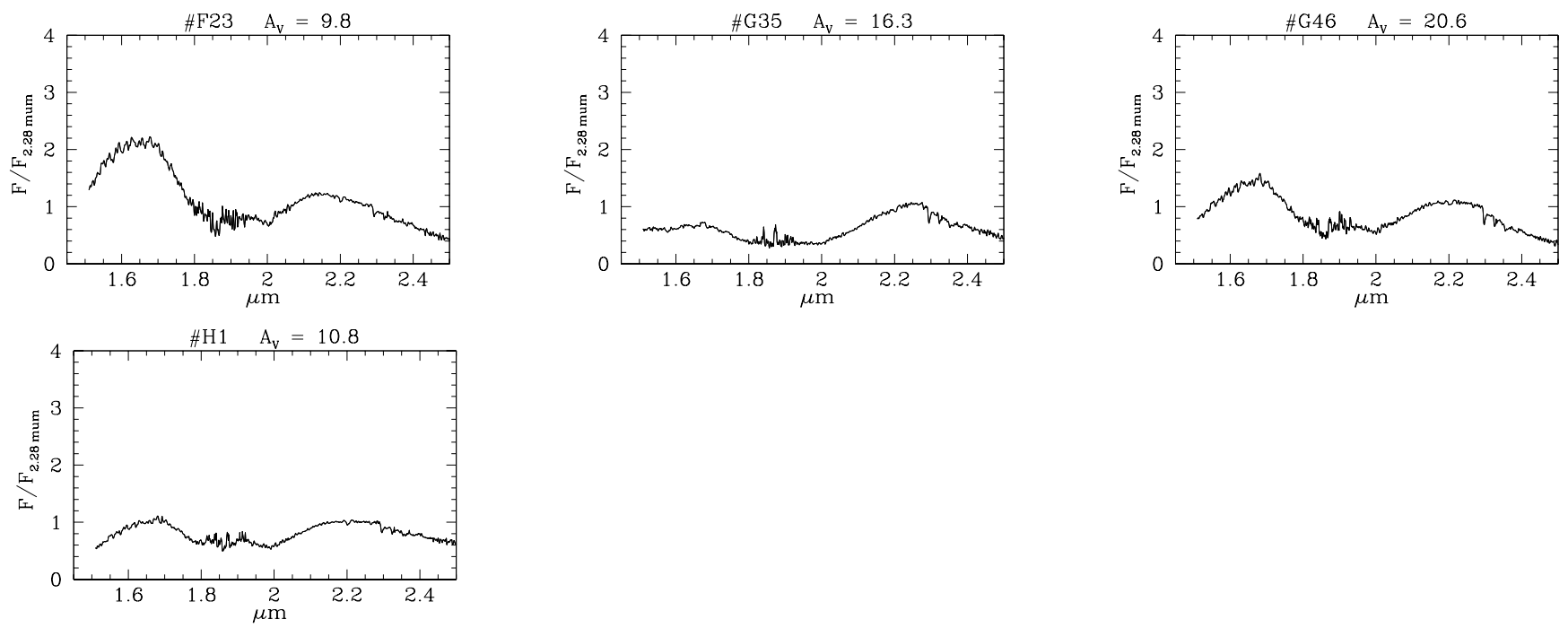

Fig. B.5. continued.
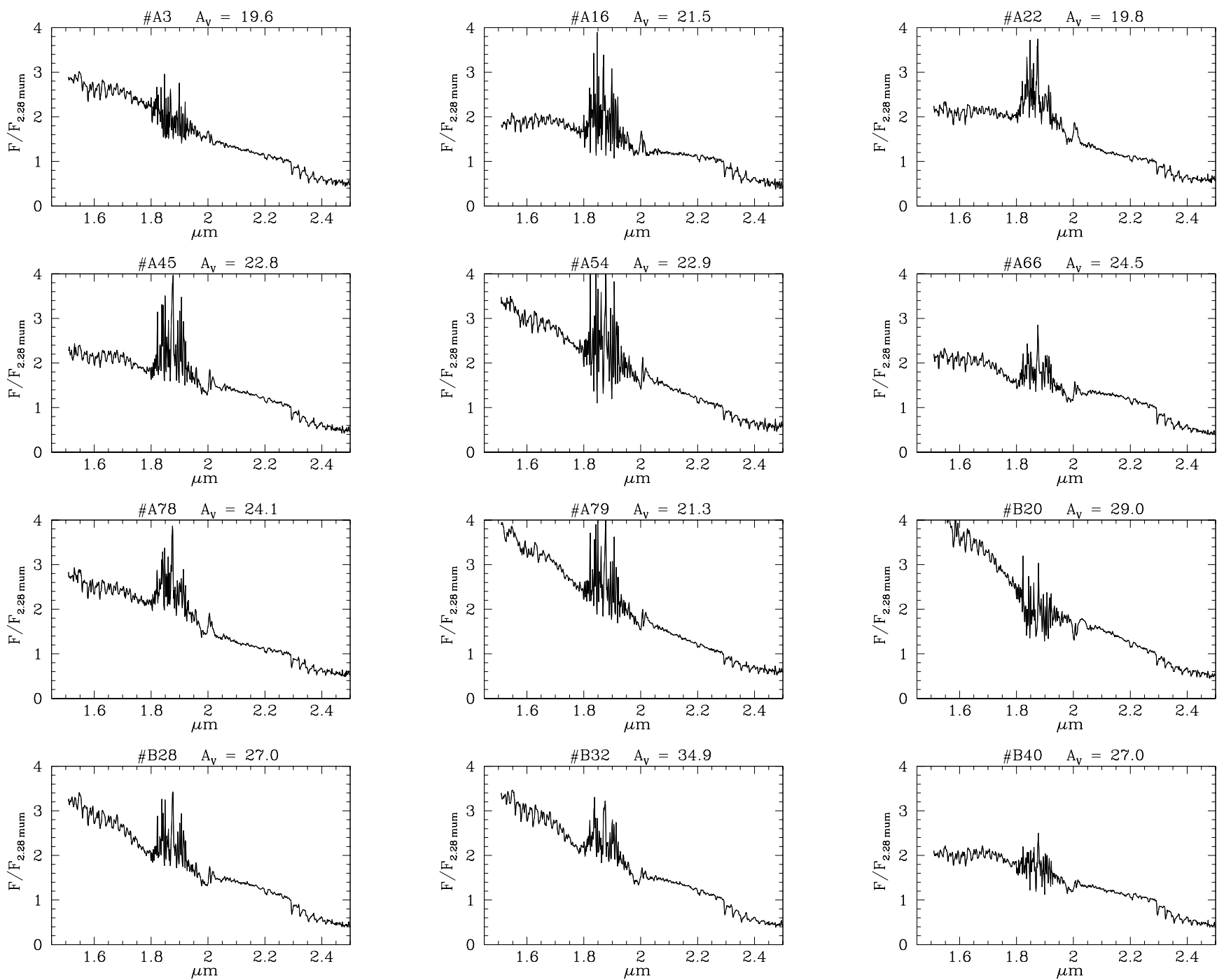

Fig. B.6. Red giant candidates. 


\section{References}

Alard, C., Blommaert, J. A. D. L., Cesarsky, C., et al. 2001, ApJ, 552, 289

Alcock, C., Allsman, R. A., Alves, D. R., et al. 2000, AJ, 119, 2194

Aringer, B., Kerschbaum, F., \& Hron, J. 2000, in The 2nd ISO workshop on analytical spectroscopy, ed. A. Salama, M. F. Kessler, K. Lech, \& B. Schulz, ESA-SP, 456

Aringer, B., Jørgensen, U. G., Schultheis, M., et al. 2002, in Determining Stellar parameters for red giants, IAU Symp. 210, Uppsala, Sweden

Bessell, M. S., Brett, J. M., Scholz, M., \& Wood, P. R. 1991, A\&ASS, 89, 335

Bessell, M. S., Scholz, M., \& Wood, P. R. 1996, A\&A, 307, 481

Blommaert, J. A. D. L., van der Veen, W. E. C. J., van Langevelde, H. J., \& Habing, H. J. 1998, A\&A, 329, 991

Catchpole, R. M., Whitelock, P. A., \& Glass, I. S. 1990, MNRAS, 247, 479

Cesarsky, C., Abergel, A., Agnese, P., et al. 1996, A\&A, 315, L32

Felli, M., Comoretto, G., Testi, L., Omont, A., \& Schuller, F. 2000, A\&A, 362, 199

Felli, M., Testi, L., Schuller, F., \& Omont, A. 2002, A\&A, 392, 971

Feuchtinger, M. U., Dorfi, E. A., \& Höfner, S. 1993, A\&A, 273, 513

Figer, D. F. 2002, in A Massive Star Odyssey, from Main Sequence to Supernova, IAU Symp. 212, ed. K. A. van der Hucht, \& C. Esteban [astro-ph/0207300]

Fluks, M. A., Plez, B., Thé, P. S., et al. 1994, A\&AS, 105, 311

Freeman, K., Bland-Hawthorn, J. 2003, ARA\&A, submitted [astro-ph/0208106]

Frogel, J. A., \& Whitford, A. E. 1987, ApJ, 320, 199

Frogel, J. A. 1988, ARA\&A, 26, 51

Frogel, J. A., Tiede, G. P., \& Kuchinski, L. E. 1999, AJ, 117, 2296

Frogel, J. A., Stephens, A., Ramírez, S. V., \& DePoy, D. L. 2001, AJ, 122,1896

Glass, I. S., Catchpole, R. M., \& Whitelock, P. A. 1987, MNRAS, 227, 373

Glass, I. S. 1999, Handbook of infrared astronomy (Cambridge University Press)

Glass, I. S., Ganesh, S., Alard, C., et al. 1999, MNRAS, 308, 127

Glass, I. S., Matsumoto, S., Carter, B. S., \& Sekiguchi, K. 2001, MNRAS, 321, 77

Glass, I. S., \& Schultheis, M. 2002, MNRAS, 337, 519

Gómez, M., \& Mardones, D. 2003, AJ, submitted [astro-ph/0301081]

Greene, T. P., \& Lada, C. J. 1996, AJ, 112, 2184

Habing, H. J., Tignon, J., \& Tielens, A. G. G. M. 1994, A\&A, 286, 523

Habing, H. J. 1996, A\&AR, 7, 97

Haywood, M. 2001, MNRAS, 325, 1365

Hennebelle, P., Pérault, M., Teyssier, D., \& Ganesh, S. 2001, A\&A, 365,598

Höfner, S. 1999, A\&A, 346, L9

Houdashelt, M. 1996, PASP, 108, 828
Ibata, R. A., \& Gilmore, G. F. 1995, MNRAS, 275, 591

Jeong, K. S., Winters, J. M., Le Bertre, T., et al. 2002, in Mass-losing Pulsating stars and their Circumstellar Matter, ed. Y. Nakasa, M. Honma, \& M. Sekiin

Josselin, E., Blommaert, J. A. D. L., Groenewegen, M. A. T., \& Li, F. L. 2000, A\&A, 357, 225

Kurucz, R. 1993, CDrom Number 13 (provided by the author)

Lançon, A., \& Rocca-Volmerange, B. 1992, A\&AS, 96, 593

Lançon, A., \& Wood, P. R. 2000, A\&AS, 146, 217 (LW)

Le Bertre, T., \& Winters, J. M. 1998, A\&A, 334, 173

Loup, C., Groenewegen, M. A. T., Cioni, M. R., et al. 2003, A\&A, submitted

Mathis, J. S. 1990, ARA\&A, 28, 37

Mezger, P. G., Dusch, W. J., \& Zylka, R. 1996, A\&AR, 7, 289

McWilliam, A., \& Rich, R. M. 1994, ApJS, 91, 749

Minniti, D., Olszewski, E. W., Liebert, J., et al. 1995, MNRAS, 277, 1293

Molla, M., Ferrini, F., \& Gozzi, G. 2000, MNRAS, 316, 345

Ojha, D. K., Omont, A., Schuller, F., et al. 2003, A\&A, 403, 141 (OOS)

Omont, A., Ganesh, S., Alard, C., et al. 1999, A\&A, 348, 755

Omont, A., Gilmore, G., Alard, C., et al. 2003, A\&A, 403, 975

Origlia, L., Moorwood, A. F. M., \& Oliva, E. 1993, A\&A, 280, 536

Origlia, L., \& Ferraro, F. R. 2002, ApJ, 571, 458

Ortiz, R., Blommaert, J. A. D. L., Copet, E., et al. 2002, A\&A, 388, 279

Philipp, S., Zylka, R., Mezger, P. G., et al. 1999, A\&A, 348, 768

Ramírez, S. V., DePoy, D. L., Frogel, J. A., Sellgren, K., \& Blum, R. D. 1997, AJ, 113, 1411 (RDF)

Ramírez, S. V., Sellgren, K., Carr, J. S., et al. 2000a, ApJ, 537, 205

Ramírez, S. V., Stephens, A. W., Frogel, J. A., \& DePoy, D. L. 2000b, AJ, 120, 833 (RSFD)

Rich, R. M. 1988, AJ, 95, 828

Schuller, F., Ganesh, S., Messineo, M., et al. 2003, A\&A, 403, 955

Schuller, F., Ph.D. Thesis

Schultheis, M., Ganesh, S., Simon, et al. 1999, A\&A, 349, L69

Schultheis, M., \& Glass, I. S. 2001, MNRAS, 327, 1193

Schultheis, M., Parthasarathy, M., Omont, A., et al. 2002, A\&A, 386, 899

Sedlmayr, E. 1994, in Molecules in the Stellar Environment, ed. U. G. Jorgensen (Berlin: Springer), 163

Serabyn, E., \& Morris, M. 1996, Nature, 382, 602

Terndrup, D. M. 1988, AJ, 96, 884

Tiede, G. P., Frogel, J. A., \& Terndrup, D. M. 1996, AJ, 110, 2788

van Loon, J. Th., Gilmore, G. F., Omont, A., et al. 2003, MNRAS, 338, 857

Vassiliadis, E., \& Wood, P. R. 1993, ApJ, 413, 641

Whitelock, P. A., Menzies, J. W., Feast, M. W., et al. 1994, MNRAS, 267, 711

Whitford, A. E. 1978, ApJ, 226, 777

Wood, P. R., Habing, H. J., \& McGregor, P. J. 1998, A\&A, 336, 925 